\title{
Star Formation Histories of Dwarf Galaxies from the Colour-Magnitude Diagrams of Their Resolved Stellar Populations
}

\author{
Michele Cignoni ${ }^{1,2}$ and Monica Tosi ${ }^{2}$ \\ ${ }^{1}$ Astronomy Department, Bologna University, Via Ranzani 1, 40127 Bologna, Italy \\ ${ }^{2}$ Osservatorio Astronomico di Bologna, INAF, Via Ranzani 1, 40127 Bologna, Italy \\ Correspondence should be addressed to Michele Cignoni, michele.cignoni@unibo.it
}

Received 5 May 2009; Accepted 12 August 2009

Academic Editor: Ulrich Hopp

Copyright ( $) 2010$ M. Cignoni and M. Tosi. This is an open access article distributed under the Creative Commons Attribution License, which permits unrestricted use, distribution, and reproduction in any medium, provided the original work is properly cited.

\begin{abstract}
In this tutorial paper we summarize how the star formation (SF) history of a galactic region can be derived from the colourmagnitude diagram (CMD) of its resolved stars. The procedures to build synthetic CMDs and to exploit them to derive the SF histories (SFHs) are described, as well as the corresponding uncertainties. The SFHs of resolved dwarf galaxies of all morphological types, obtained from the application of the synthetic CMD method, are reviewed and discussed. To summarize: (1) only early-type galaxies show evidence of long interruptions in the SF activity; late-type dwarfs present rather continuous, or gasping, SF regimes; (2) a few early-type dwarfs have experienced only one episode of SF activity concentrated at the earliest epochs, whilst many others show extended or recurrent SF activity; (3) no galaxy experiencing now its first SF episode has been found yet; (4) no frequent evidence of strong SF bursts is found; (5) there is no significant difference in the SFH of dwarf irregulars and blue compact dwarfs, except for the current SF rates. Implications of these results on the galaxy formation scenarios are briefly discussed.
\end{abstract}

\section{Introduction}

Dwarf galaxies are the most diffused type of galaxies in the Universe and were probably even more numerous in the past, when they might have contributed to the population of blue systems overabundant in deep galaxy counts (e.g., $[1,2])$ and more likely to the assembling of larger baryonic systems. In spite of having received less attention than spiral and elliptical galaxies, dwarf galaxies have probably more cosmological relevance. For instance, late-type dwarfs are the preferred targets for cosmologists interested in Big Bang Nucleosynthesis, because their low metal and helium contents allow the derivation of the primordial helium abundance from HII regions spectra with minimum extrapolation (e.g., [3-5]). Moreover, their low metallicity and high gas content make them apparently less evolved than spirals and ellipticals, thus more similar to what primeval galaxies may have been.
One of the main cosmological interests is related to the possibility that today's dwarfs are the survivors of the building blocks of massive galaxies. Cold Dark Matter (CDM) cosmology predicts that dwarf systems are the first ones to form after the Big Bang, since only dark matter halos of mass smaller than $10^{8} M_{\odot}$ are able to condense from primordial density perturbations. In this framework, more massive systems are assembled by subsequent merging of these protogalactic fragments (the hierarchical formation scenario; e.g., $[6,7])$, and dwarfs have a pivotal role in the evolution of massive galaxies.

Observations show that galaxies merge in the local Universe and that big galaxies accrete their satellites. We know the cases of the Magellanic Stream and of other streams connected to the Sagittarius dwarf spheroidal (dSph) and other satellites falling on the Milky Way (e.g., $[8,9])$. Andromeda is quite similar in this respect (e.g., $[10-12]$ ), with streams and clumps just as, or even more than, our own 
Galaxy. The question is whether big galaxies form only by successive mergers of smaller building blocks, as proposed by the hierarchical formation scenario, or satellite accretion is a frequent but not necessary and dominant event, consistent with a downsizing formation scenario. Downsizing [13] in principle does not concern the hierarchy or the epoch of galaxy formation, it simply reflects the observational evidence that the bulk of stars in more massive galaxies formed earlier and at a higher rate than those in less massive systems. If mechanisms are found allowing for these star formation properties in the bottom-up scenario (e.g., [1416]), then downsizing is not incompatible either with CDM or with the hierarchical scenario. However, downsizing is often seen as the alternative to hierarchical formation, replacing in this role the monolithical scenario, where each galaxy forms from the collapse (dissipative or dissipationless) of its protogalactic gas cloud. In the monolithical scenario more massive galaxies form much earlier than less massive ones for simple gravitational arguments [17], with timescales for the collapse of the protogalactic cloud originally suggested to be of the order of $100 \mathrm{Myr}$ and now more often considered of the order of 1 Gyr.

One of the effective ways to check whether or not big galaxies are made only by successive accretions of satellites like the current ones is to observe the resolved stellar populations of massive and dwarf systems and compare their properties with each other. If chemical abundances, kinematics, and star formation histories of the resolved stars of massive galaxies are all consistent with those of dwarf galaxies, then the former can be the result of successive merging of the latter; otherwise, either satellite accretion is not the only means to build up spirals and ellipticals or the actual building blocks are not alike today's dwarfs.

An updated review of the chemical, kinematical, and star formation properties of nearby dwarfs can be found in [18]. In this tutorial paper, we describe how the star formation history of a galactic region can be derived from the colourmagnitude diagram of its resolved stars, and we summarize what people have learnt on the SFHs of dwarf galaxies from the application of the most popular approach based on the CMD. In Section 2 we introduce the method; in Section 3 we describe in detail procedures and uncertainties; in Section 4 we report on the results of its application on the SFH of dwarf galaxies. A discussion on how these results may affect our understanding of galaxy evolution which is presented in Section 5.

\section{Star Formation Histories from Colour-Magnitude Diagrams: The Method}

The need for understanding the star formation histories of dwarf galaxies was recognized long ago and over the years many approaches have been followed to infer the SFH of different galaxies. Among the many studies devoted to the field, we recall the seminal paper [19] and the extensive and detailed analyses performed by Gallagher, Hunter and collaborators (see $[20,21]$, and references therein), who used various indicators to estimate the star formation rates at different epochs of large samples of dwarfs. The quantum leap in the field occurred two decades ago, when the power and resolution of new generation telescopes and detectors allowed people to resolve and measure individual stars even in the crowded fields of external galaxies and to draw their CMDs. The CMD of a stellar system is in fact the best information desk on the system evolution, because it preserves the imprinting of all the relevant evolution parameters (age, mass, chemical composition, initial mass function).

Twenty years ago, stellar age dating was done with isochrone fitting, a handy tool for simple stellar populations, such as those of stellar clusters, but inadequate to interpret the composite populations of galaxies, where many subsequent generations of stars, with possibly different initial mass function, metallicity, reddening, and distance, contribute to the morphology of the observational CMD. With CCD detectors and new reduction packages for PSF fitting photometry allowing for the first time to measure accurately individual stars in Local Group (LG) galaxies, the time had come to develop a reliable tool to quantitatively derive their SFHs. The best tool is based on CMDs, and is the extrapolation of the standard isochrone fitting method to the complicated CMDs of composite stellar populations: the synthetic CMD method.

2.1. Building a Synthetic Population. The synthetic CMD method allows us to derive all the SFH parameters within the lookback time reached by the available photometry. To do this, a synthesizer works with classical ingredients:

(i) star formation law and rate, $\operatorname{SFR}(\mathrm{t})$, which regulate the astrated mass at each time $t$;

(ii) initial mass function (IMF), which gives the number $N$ of stars in each generation per unit stellar mass interval, a useful form is a power-law:

$$
d N \propto M^{-\alpha} d M
$$

the IMF is usually assumed to be independent of time;

(iii) chemical enrichment due to the galaxy chemical evolution, the metallicity of the gas from which stars form changes with time, this is described by an agemetalicity relation (AMR) $Z(t)$;

(iv) stellar evolution tracks, giving the temperature and luminosity of stars of given mass and metallicity at any time after their birth;

(v) stellar atmosphere models, to transform the bolometric magnitudes and temperatures into the observational plane;

(vi) binary fraction and mass ratio.

The standard procedure is the following. Using a Monte Carlo algorithm, masses and ages are extracted according to the IMF and the SF law (e.g., constant or exponentially decreasing with time, proportional to some power of the gas density, etc.). The metallicity follows suitable AMRs. 
The extracted synthetic stars are placed in the CMD by interpolation among the adopted stellar evolution tracks of the assumed metallicity. In order to take into account the presence of unresolved binary stars, a chosen fraction of stars are assumed to be binaries and coupled with a companion star. The fake population is put at the distance of the galaxy we want to analyze, simultaneously correcting for reddening and extinction. Finally, photometric errors, incompleteness, and blending factors, as accurately estimated from artificial star tests on the actual photometric images of the examined field, are applied to the synthetic CMD.

Different combinations of the parameter choices provide the whole variety of CMDs observed in real galaxies. As an example, Figure 1 shows the CMDs resulting from 6 representative, although simplistic, cases.

Figures 1(a)-1(f) illustrate the effect of different SFHs on the synthetic CMD of a hypothetical galactic region with a number of resolved individual stars, photometric errors, as well as blending and incompleteness factors typical of a region in the SMC imaged with HST/WFPC2. Figures 1(a)1(c) show examples of CMDs typical of late-type galaxies, with ongoing or recent star formation activity. If the SFR has been constant for all the galaxy lifetime, the CMD of the region is expected to have the morphology of Figure 1(b), with a prominent blue plume mostly populated by mainsequence (MS) stars and an equally prominent red plume resulting from the overposition of increasingly bright and massive stars in the red giant branch (RGB), asymptotic giant branch (AGB), and red supergiant phases. At intermediate colours, for decreasing brightness, stars in the blue loops, red clump, and subgiant phases are visible, as well as the stars at the oldest MS turnoff (MSTO) and on the faint MS of low mass stars. Stars of all ages are present, from those as old as the Hubble time to the brightest ones a few tens Myr old.

If we leave the SFH unchanged except for the addition of a burst ten times stronger concentrated in the last $20 \mathrm{Myr}$, the CMD (Figure 1(a)) has a much brighter and more populated blue plume, now containing many stars a few Myr old. In Figure 1(c) the same constant SFR as in the first case is assumed, but with a quiescent interval between 3 and 2 Gyrs ago: a gap is clearly visible in the CMD region corresponding to isochrones with the age of the missing stars.

The three bottom panels of Figure 1 show CMDs typical of early-type galaxies, whose SF activity is concentrated at earlier epochs. If only one SF episode has occurred from 13 to $10 \mathrm{Gyr}$ ago, with a constant metallicity $Z=0.004$ as in Figures $1(\mathrm{a})-1(\mathrm{c})$, the resulting CMD is shown in Figure 1(e). If the SF has occurred at the same epoch, but with a metallicity ten times lower, the evolutionary phases in the resulting CMD (Figure 1(f)) have colours and luminosities quite different from the previous case. Finally, Figure 1(d) shows the case of two bursts, the first from 13 to $11 \mathrm{Gyr}$ ago and the second from 5 to $4 \mathrm{Gyr}$ ago. The gap corresponding to the quiescent interval is visible in the CMD although not as much as the more recent gap of Figure 1(c).

Once a synthetic CMD is built, the challenging part of the method is the comparison with the observational CMD. The best values of the parameters (IMF, AMR, SFR, binary fraction, reddening, and distance modulus) are found by selecting the cases providing synthetic CMDs with morphology, colours, number of stars in the various evolutionary phases, and luminosity functions consistent with the observed ones. Independently of the specific method, any approach is unavoidably statistical and does not provide a unique solution for the SFH of the examined region. Nevertheless, the synthetic CMD method is quite powerful, thanks to the wealth of independent constraints available in a good $\mathrm{CMD}$, and it strongly reduces the range of possible scenarios.

In what follows, we will describe what are the major uncertainties in the method. Before diving into details, however, it is crucial to understand which parts of a CMD are the most reliable clocks.

2.2. Stellar Ages from a CMD. What can be learnt from a $\mathrm{CMD}$ ? All the evolutionary sequences are witness of the same $\mathrm{SFH}$, but some sequences are specifically sensitive to age more than to any other ingredient (e.g., metallicity, convection, etc.). In order to track the history of a galaxy, it is necessary to select the safest age indicators. Because different evolutionary phases populate different $\mathrm{CMD}$ regions, one must then know which parts of the CMD are more informative.

The best indicators share a useful feature: the age is related to the luminosity, which depends on the burning rate and on the available fuel. The MS is the archetype of this class of phases, since in this stage the stars obey a mass-luminosity relation $L \propto M^{n}$ (where $n$ varies from 3 to 4 ). This relationship has strong implications. Adopting a mean value $n=3.5$ and considering that the available fuel is proportional to the stellar mass $M$, the time spent in MS is proportional to $M^{-2.5}$ : massive stars live for short times (50 Myr for a $8 M_{\odot}$ ), mapping only the recent SFH, while objects smaller than $1.5 M_{\odot}$ can survive for many Gyr, mapping the recent as well as the ancient star formation history. From the point of view of the CMD, this one-to-one correspondence of luminosity and mass/age allows, for a given metallicity, a direct conversion of the MS information into the SFH.

Beyond the MS, a mass-luminosity relation does not hold anymore, and the luminosity is rather sensitive to the core mass growth. The phases between the MS and the red giant are so fast (thermodynamical evolution) that the probability of observing their stars is low (compared to that for nuclear phases). This causes the so-called Hertzsprung gap, that is, the observed lack (or paucity) of stars in the evolutionary phase right after the MS. However, for stars smaller than about $2-2.5 M_{\odot}$, the evolutionary times are long enough (because the degeneracy pressure prevents a rapid core contraction) to define another useful age indicator: the subgiant branch (SGB). Like the MS turn-off, the SGB fades as the the age increases.

Later evolutionary phases, namely, the RGB, the horizontal branch (HB), the red clump (RC), and the asymptotic giant branch, with the exception of the blue loop (BL) stage, are questionable age indicators. In fact, the CMD position of such objects mostly reveals the age through the colour, which can be influenced by several factors. As an example, aging the stars makes the RGB redder, but theoretical uncertainties like the colour transformations and the super-adiabatic convection can lead to higher colour shifts. The RGB is 


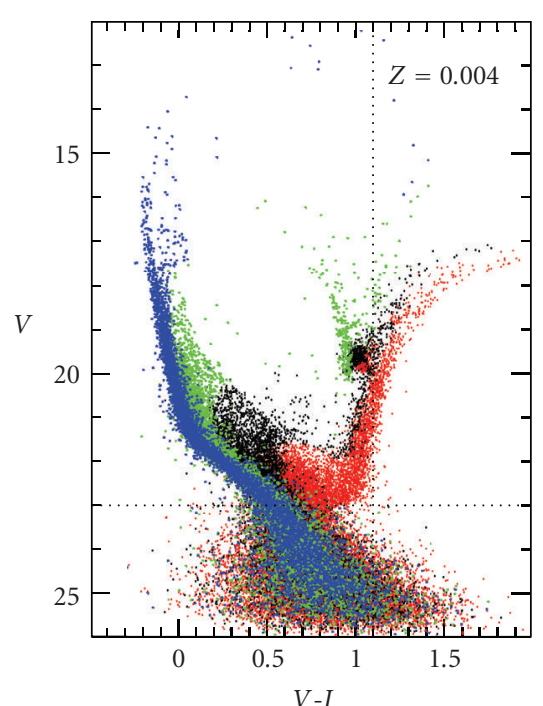

(a)

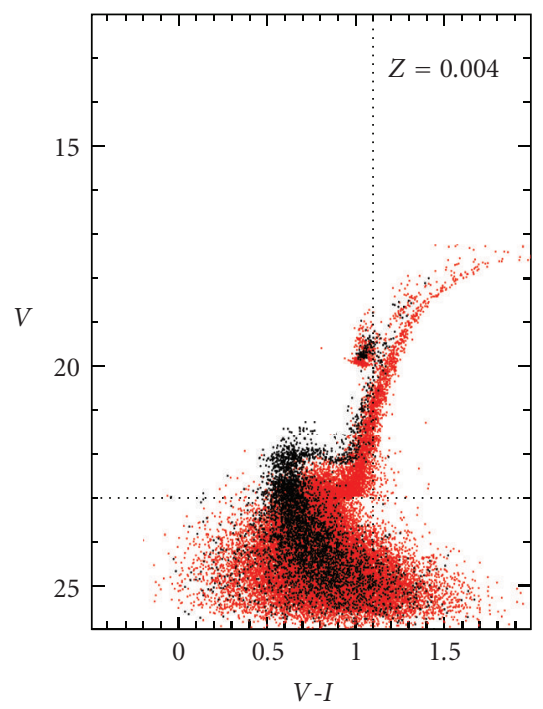

(d)

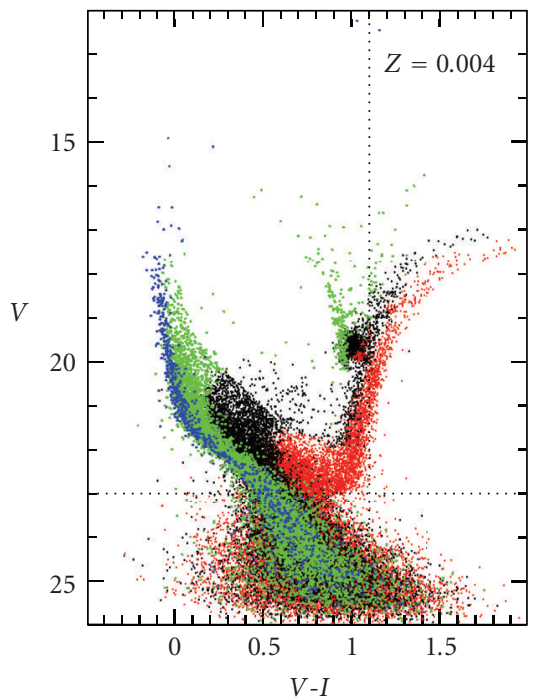

(b)

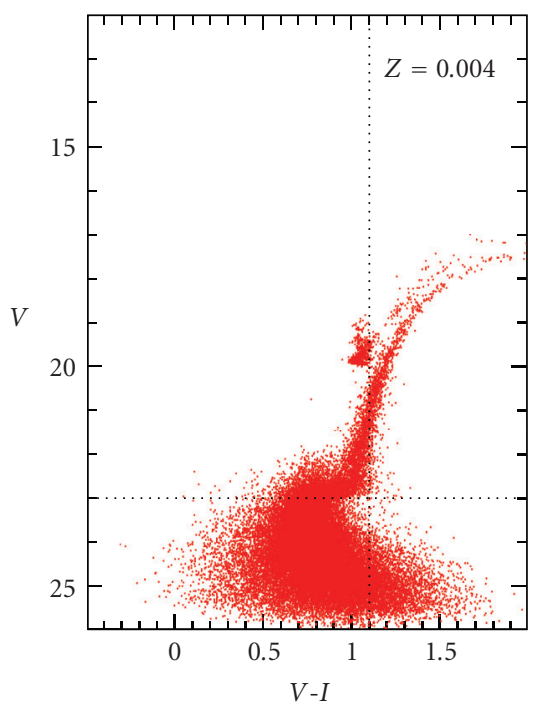

(e)

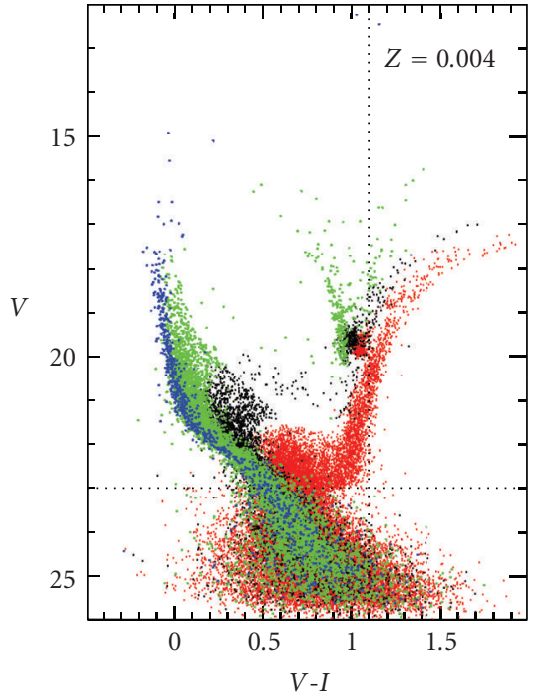

(c)

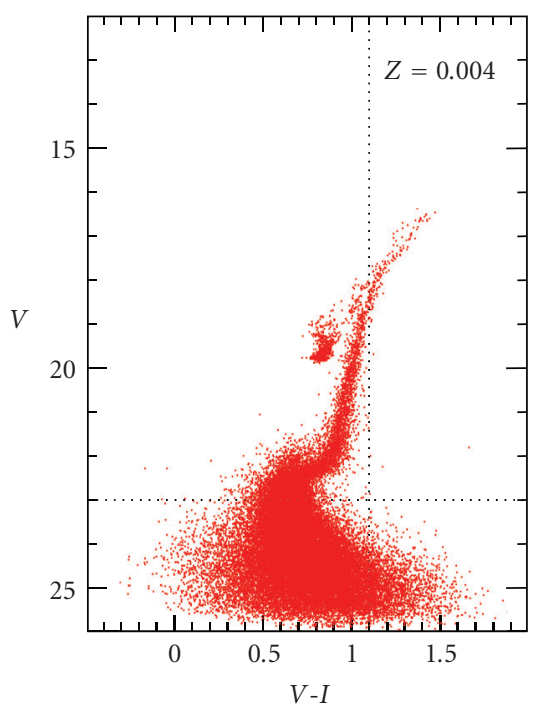

(f)

Figure 1: The effect of the SFH on the theoretical CMD of a hypothetical galactic region with $(m-M)_{0}=19, E(B-V)=0.08$, and with the photometric errors and incompleteness typical of HST/WFPC2 photometry. All the shown synthetic CMDs contain 50000 stars and are based on the Padova models [22, 23] with the labelled metallicities. (b) The case of an SFR constant from 13 Gyr ago to the present epoch. (a) The effect of adding a burst 10 times stronger in the last 20 Myr to the constant SFR. The CMD has a much brighter and thicker blue plume. (c) The same constant SFR as in the first case, but with a quiescence interval between 3 and 2 Gyrs ago; a gap appears in the CMD region corresponding to stars 2-3 Gyr old, which are completely missing. (e) SF activity only between 13 and $10 \mathrm{Gyr}$ ago with $Z=0.004$. (f) SF activity only between 13 and $10 \mathrm{Gyr}$ ago with $Z=0.0004$ : notice how colour and luminosity of turnoff, subgiant, and red giant branches differ from the previous case. (d) SF activity between 13 and 11 Gyr ago, followed by a second episode of activity between 5 and 4 Gyr ago: a gap separates the two populations in the CMD, but less evident than in (c) case, when the quiescent interval was more recent.

undoubtedly more sensitive to the metallicity. On the other hand, all these phases are indisputable signature of stars older than a limiting age and extremely useful in the age dating of galaxies too distant to have the MSTO reachable by any photometry: HB stars are always older than 10 Gyr, RGBs at least 1-2 Gyr old, and AGBs older than 100 Myr. Moreover, as thoroughly discussed by Greggio [24], the relations existing between the number counts of post-MS stars and their mass helps in constraining the SFH.
Among core helium burning stars, the $\mathrm{HB}$ and the RC phases are composed by stars of initial mass smaller than about 2 solar masses, whose luminosity depends on the helium core mass and is quite independent of the stellar mass. In particular, the HB colour frequently shows a correlation with metallicity (the "first parameter"), while age is only one of the possible secondary parameters. Quite different is the behavior of intermediate mass stars (over $2 M_{\odot}$ ): during the core helium burning these objects describe 
a large loop in colour (the so-called blue loops) and their luminosities are critically sensitive to the mass; this is because the core mass is connected to the extension of the convective cores in the previous MS structures. Thanks to this feature, for ages of 100-500 Myr, the luminosity of the loops fades with age and the $\mathrm{BL}$ is an excellent age indicator.

After the core helium burning phase, low and intermediate mass stars experience the AGB phase. As for the RGB, a relation between the luminosity and the core mass holds. In addition, many phenomena occur (e.g., mixing and extra mixing of convective layers, thermal pulses, etc.) which are not yet understood in detail, and this leaves considerable uncertainty.

The very first and the final stages of stellar evolution, namely, the pre-main sequence (PMS) and the white dwarf (WD) regimes, also deserve some comments. As for the former, prior to reaching the MS, the star's energy source is a contraction on thermodynamical timescales (tenths of Myr). While aging, PMS stars fade and become hotter. Figure 2 shows the HST/ACS images and the corresponding CMDs of two star forming regions in the Small Magellanic Cloud, NGC602 (left-hand panels, [25]) and NGC346 (right-hand panels, [26]). The well-defined sequence well separated from the canonical main sequence, which appears on the righthand side of these CMDs, is composed by PMS stars. Were not this phase affected by several theoretical and observational uncertainties, the PMS would be a powerful clock for the most recent Myrs (see, e.g., [27], and references therein).

On the other hand, WDs represent the final fate of all stars with masses below $8 M_{\odot}$. These stars share a useful feature: the peak of the WD luminosity function fades with age. Unfortunately, the presence of theoretical uncertainties (e.g., crystallization processes, nuclear reaction rates, convection, mass loss, and initial chemical composition) together with an intrinsic low luminosity $\left(10<M_{V}<18\right)$ tend to invalidate its reliability.

In conclusion, MS and SGB stars are certainly the most reliable age indicators. If we add that in these phases faint objects live longer, a deeper CMD gives a better chance to robustly trace the past star formation history. It is important to underline that quantitative and qualitative indicators can be combined and typically complement each other. In other words, when a deep CMD is not available, evolved stars can be used very profitably to recover the SFH although with higher uncertainty and within shorter lookback times.

2.3. Deep is Better. The presence of a completeness limit (due to both the intrinsic crowding and distance of the examined galaxy and to the instrumental capabilities) hinders the possibility to exploit all the information contained in a CMD. To visualize this effect, we built age-frequency plots for various stellar mass ranges, assuming different completeness limits (see Figure 3) and using an artificial population generated from the Padova stellar models [22] with $Z=$ 0.004 , no binaries, and constant SFR. In order to be as general as possible, all the results are shown using absolute magnitudes. In all panels of Figure 3 we plot with different colours the fraction of stars of various mass ranges visible in the CMD above the assumed completeness limit as a function of their age.

In Figure 3(a) the completeness limit is set to $M_{V}=2.5$ : we see that on the MS only stellar masses higher than $1.5 M_{\odot}$ are brighter than this limit and usable witnesses of the last 2 Gyr. On the other hand, the long-lived nature of lower masses guarantees to trace long periods of star formation, but not the recent SFR. The explanation comes from the limit $M_{V}=2.5$ itself: it cuts off the MS, so for stars below $1.5 M_{\odot}$, we see only later (i.e., brighter) evolutionary phases, represented by the RGB and the central helium burning (the dotted lines for the mass ranges $0.6-1.0 M_{\odot}$ and 1.0-1.5 $M_{\odot}$ represent the contribution of PMS, MS, and SGB stars).

Moving the limit to $V=3.5$ (Figure 3(b)), the mass interval $1-1.5 M_{\odot}$ is visible in the CMD and informative of the star formation history between now and $\sim 8 \mathrm{Gyr}$ ago. However, only masses over 1.2-1.3 $M_{\odot}$ are on the MS. Lower masses or, equivalently, older epochs, must refer to RGB and He burning phases. The situation improves when the completeness limit is set at $M_{V}=4.5$ (Figure 3(c)), and the MS phase is visible for all stars down to $\sim 0.8 M_{\odot}$. This limit represents a very good level for studying the history of a resolved galaxy, since it guarantees age-sensitive tracers (MS and SGB stars) covering the entire Hubble time (13 Gyr).

Finally, Figure 3(d) shows the age plots for a completeness limit $M_{V}=5.5$ : at this luminosity, the zero age main sequence is reached for subsolar masses, whose lifetimes are longer than the age of the Universe. With respect to $M_{V}=4.5$ case, the advantages here are (1) a much more reliable photometry of the turn-off and SGB stars; (2) further information on the IMF, thanks to a better coverage of the lowest/faintest mass intervals, where the IMF slope may significantly change (see, e.g., $[28,29]$, and references therein); (3) a better constraint on $Z(t)$, given the mild influence of the SF law on the CMD position of low mass stars.

These results depend on the assumed chemical composition. This is important when one considers that in galaxies some chemical enrichment must always be at work. Following this paradigm, the oldest stars in a galaxy are expected to be metal poor. Changing the metallicity has two main effects on the model; namely, changes in the evolutionary lifetimes and changes in the stellar luminosity, which in turn can sensibly modify the relation between CMD and SFH. To investigate this phenomenon, in Figure 4, the frequency-age plot for the completeness limit $M_{V}=4.5$ is shown for two different metallicities, $Z=0.004$ (thick lines in the figure) and $Z=0.0004$ (thin lines). Lowering the metallicity accelerates the evolution, and the age distribution for all the mass intervals (except $0.6-1 M_{\odot}$ ) is shifted by, at least, $1 \mathrm{Gyr}$ with respect to the $Z=0.004$ cases. The age plot for masses $0.6-1 M_{\odot}$ has a different genesis: part of these stars live more than $13 \mathrm{Gyr}$, so the evolutionary effect is not visible. In contrast, the mass range $0.6-1 M_{\odot}$ emphasizes the luminosity effect: a lower metallicity pushes MS stars over the completeness limit $M_{V}=4.5$, injecting younger stars in the age distribution that now involves ages between 0 and 13 Gyr. In practice, a lower metallicity mimics what happens with a more favorable completeness limit. 


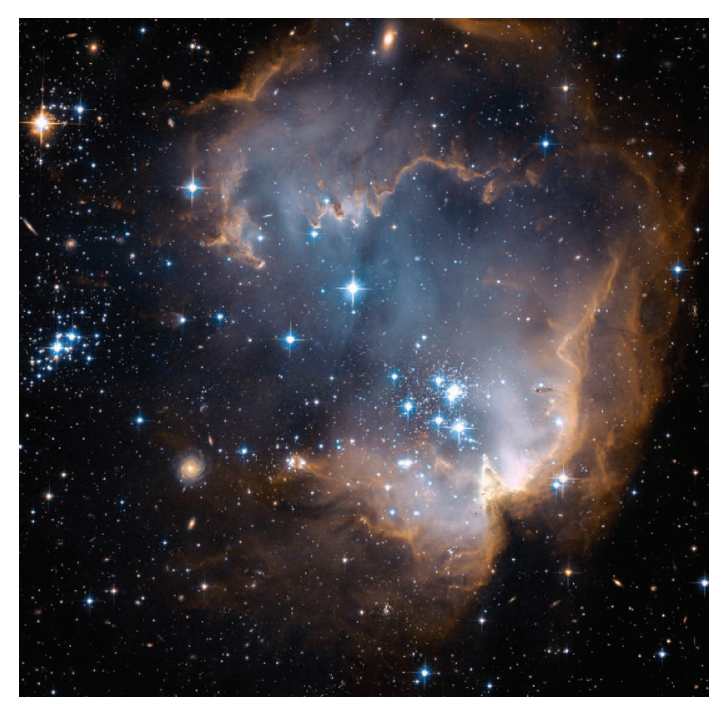

(a)

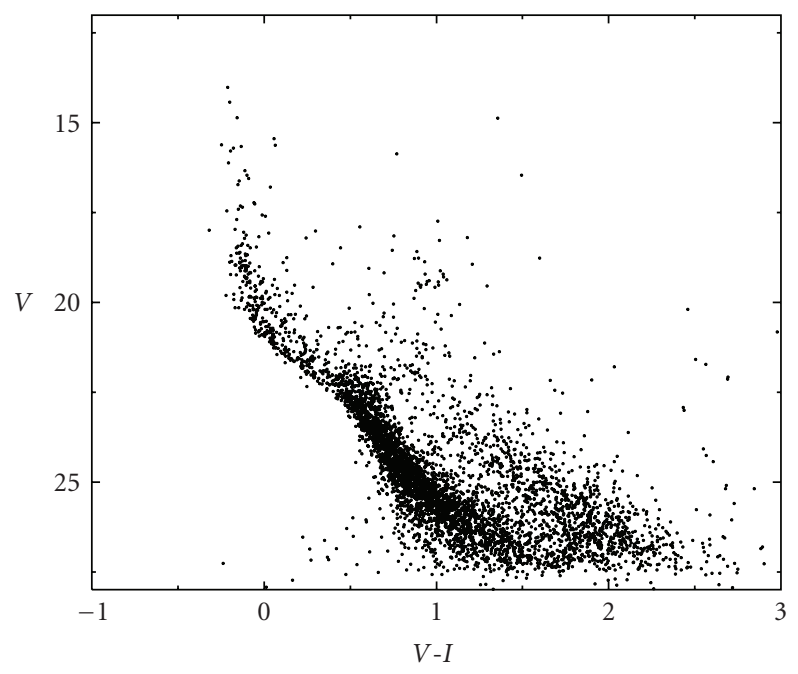

(c)

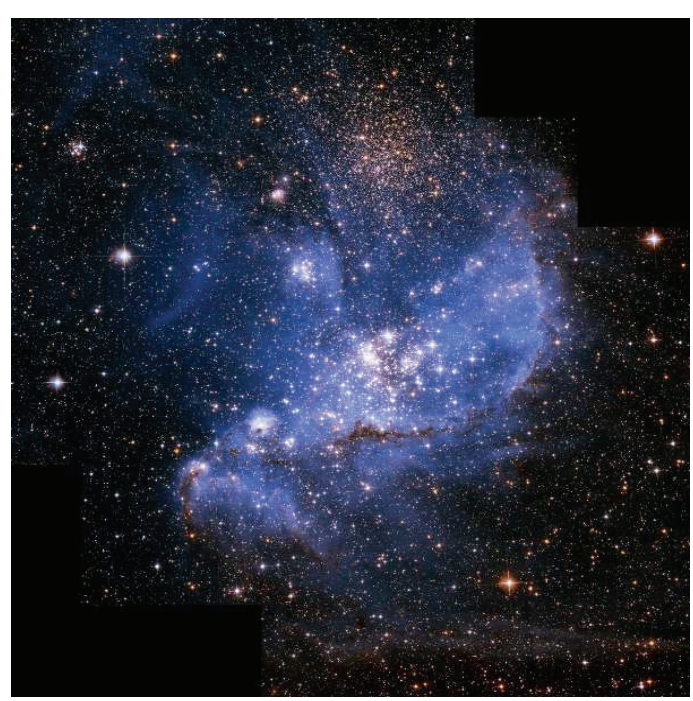

(b)

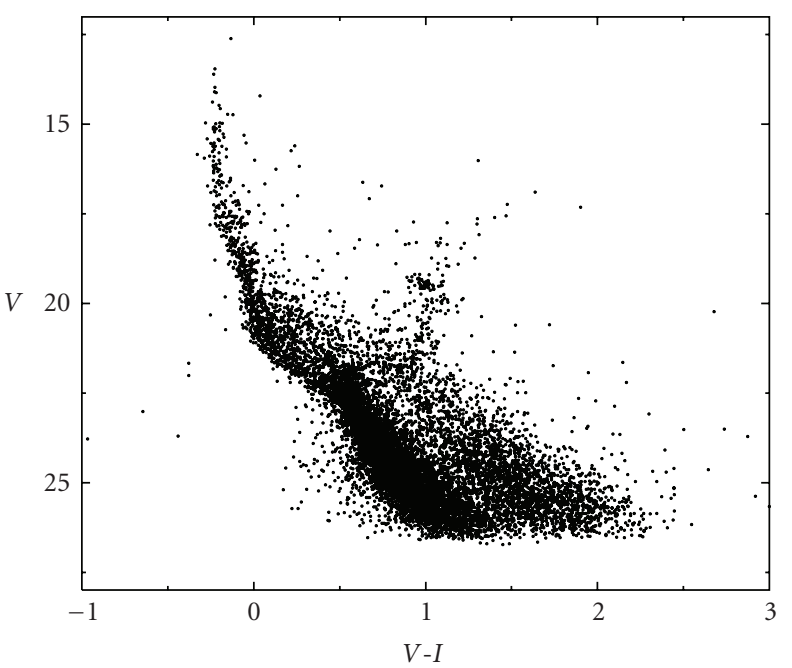

(d)

FIgURE 2: (a, b) HST/ACS true colour images of NGC602 (a), a very young cluster in the wing of the SMC and NGC346 (b), a populous young cluster in the main body of the SMC. Images credit NASA, ESA, and A. Nota (STScI, ESA). (c, d) CMD of NGC602 (c) and of NGC346 (d). PMS stars are clearly visible at the right of the Main Sequence.

This implies the following rule: in order to safely use the CMD for an estimate of the oldest star formation history we need to resolve all the stars down to $M_{V}=4.5$. Since this magnitude can be reached only in the closest galaxies, this implies that in most cases the information on the earliest SF activity is either completely lacking or very uncertain.

\section{Deducing the SFH: Guidelines}

3.1. A Changing Landscape. The first procedures to derive the $\mathrm{SFH}$ of nearby galaxies from synthetic CMDs were developed by the Bologna and the Padova groups about 20 years ago [30-34], with the latter then combining with the Canary group [35-37]. These works used luminosity functions, colour distributions and the general CMD morphology to constrain the underlying SFH. In particular, the ratio of star counts in several regions of the CMD was used to determine both the SFR and the IMF [32]. The drawback of these procedures is the lack of a robust statistical criterion to evaluate the best solution and the corresponding uncertainties. On the other hand, these authors made an optimal use of all the CMD phases and took into careful account all the properties and uncertainties of stellar evolution models, thus avoiding blind statistical approaches, which can lead to misleading results.

Later on, several methods have been proposed to statistically compare simulated and observed CMDs. In this framework, some groups have derived the SFHs of galaxies in the LG (see, e.g., [37-44]). Others (see, e.g., [45-48]) have tackled the question of the SFH in the solar neighborhood. To the same class of investigators we can assign also 


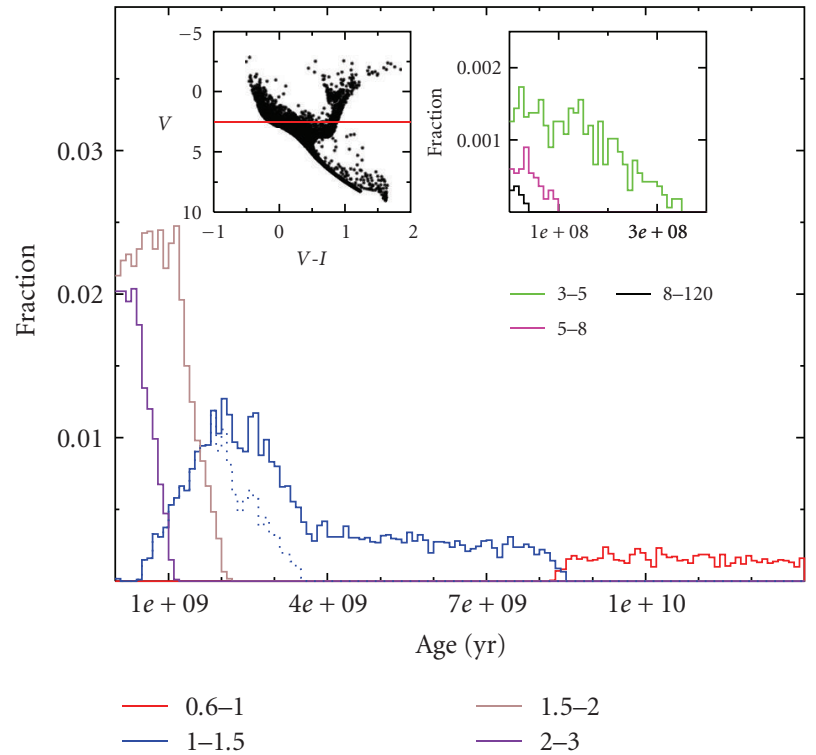

(a)

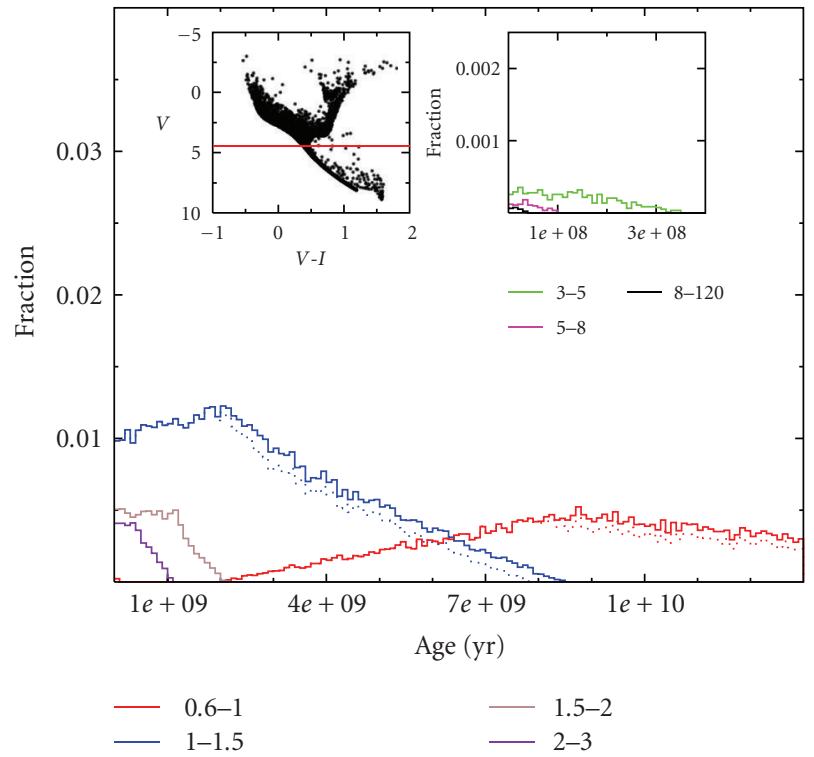

(c)

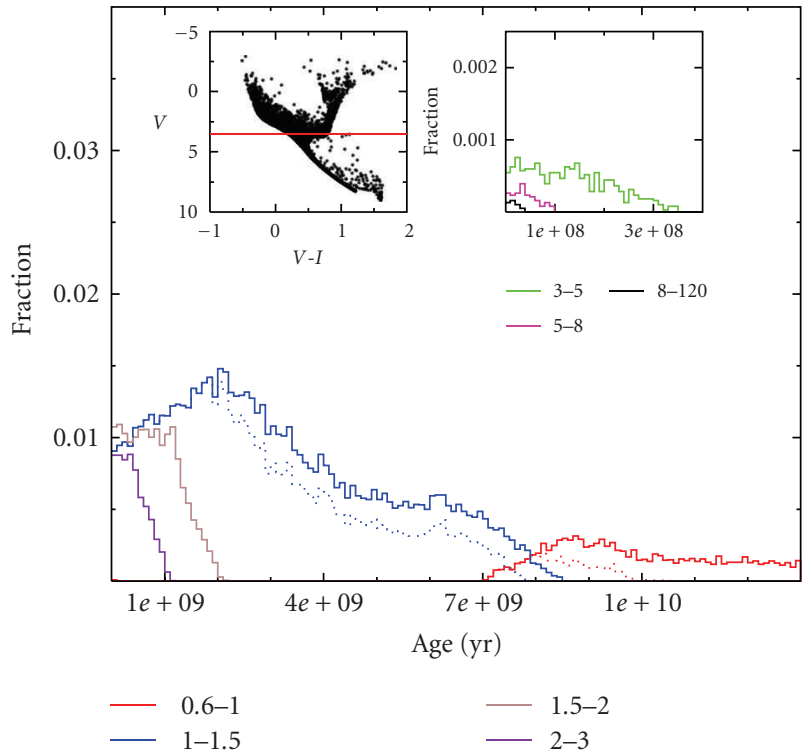

(b)

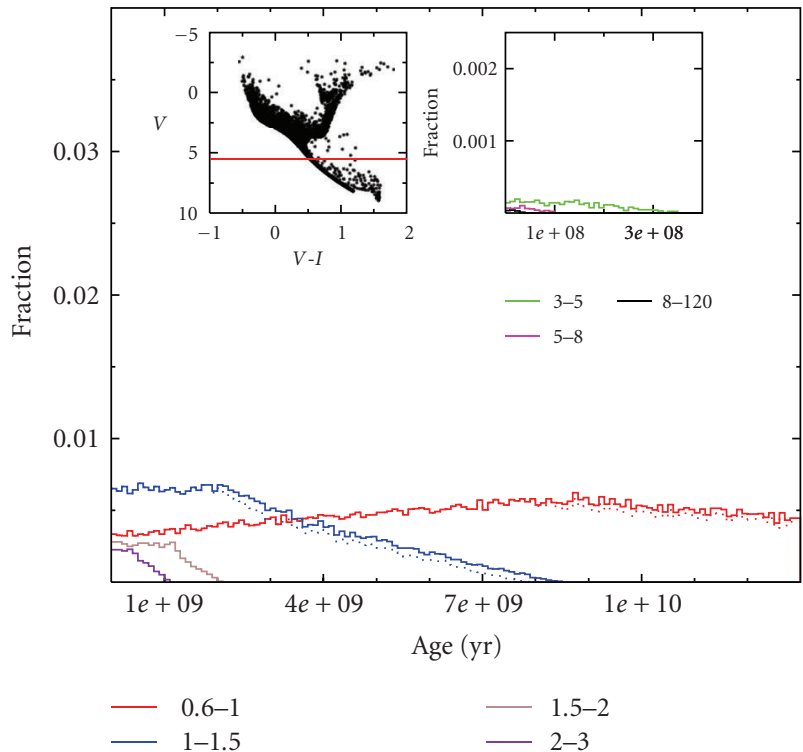

(d)

FIGURE 3: Fractional age distributions of stars generated with a constant SFR (0-13 Gyr) for four different completeness limits. In different colours (solid lines) are plotted the contributions from different mass ranges. In (a), only stars with absolute magnitude brighter than 2.5 are plotted, while in (b), (c), and (d), this limit is, respectively, $M_{V}=3.5, M_{V}=4.5$, and $M_{V}=5.5$. The dotted lines represent the contribution of PMS, MS, SGB. In each panel, the left and right subfigures show, respectively, the adopted simulated population (with the limiting magnitude marked by a red line) and a blow-up with the contribution of intermediate and massive stars in the last $300 \mathrm{Myr}$.

the study by [49] focussing on star clusters. In all these works, the emphasis is transferred from the stellar evolution properties to the problem of selecting the most appropriate model through decision making criteria: here, the likelihood between observed and model CMD is evaluated on statistical bases. There are subtle differences among different groups, reflecting how these authors define the likelihood and how they solve it. The advantages are mainly three: the possibility to exploit each star of the CMD, and not only few strategic ratios; the evaluation of the uncertainty on the retrieved
$\mathrm{SFH}$, which is robust; the explorability of a wide parameter space. However, a blind statistical approach is not risk-free. Although significant advances have been made in stellar evolution and atmosphere theories, several processes (only to cite the most infamous ones, the HB morphology, RGB and AGB features, convection in general) remain poorly understood and affect the statistical tests. If some parts of the CMDs have a low reliability and others are statistically weak, but very informative (like the helium burning loops), any blind algorithm may miss something crucial. In this case, 


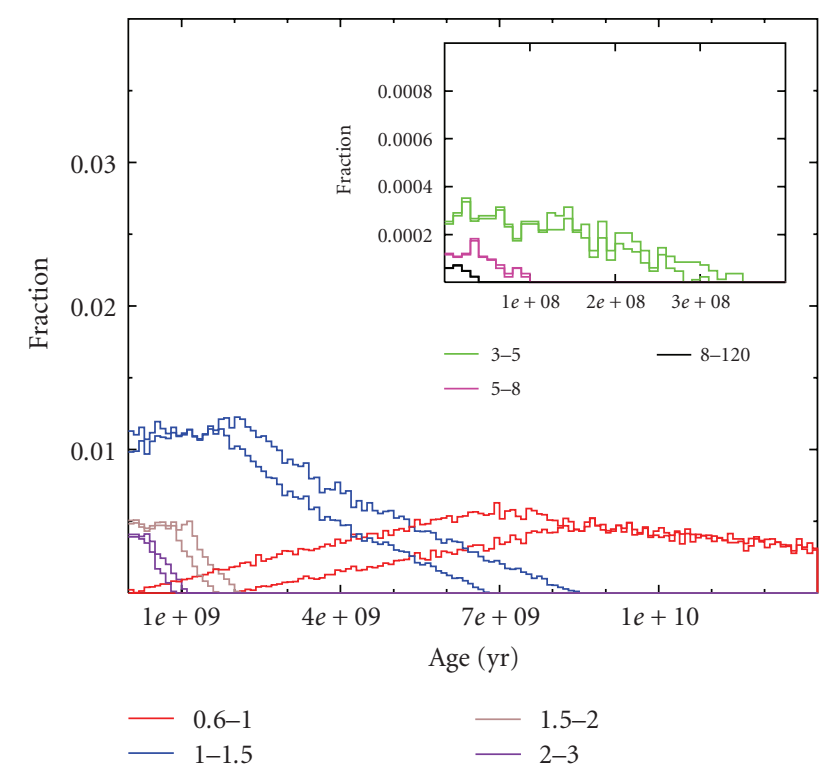

Figure 4: Fractional age distributions for stars brighter than $M_{V}=$ 4.5 at two different metallicities. The thick line represents $Z=$ 0.004 , while the thin line represents $Z=0.0004$.

a careful inspection of the CMD morphology, in particular the ratios of stellar number counts in different evolutionary phases is the irrenounceable and necessary complement to the statistical approach. Finally, whatever the adopted procedure, the absolute rate of star formation must be obtained normalizing the best model to the observed number of stars.

The major differences among the various procedures concern the approach to select the best solutions and the treatment of metallicity variations. In 2001, the predictions of the synthetic CMD method from about ten different groups were compared with each other, showing that within the uncertainties, most procedures provided consistent results (the Coimbra Experiment, see [50], and references therein).

In the following section we describe the main steps to rank the likelihood among CMDs.

3.2. To Grid or Not to Grid. In order to decide if a synthetic CMD is a good representation of the data, the observed and the model star-counts can be compared in a number of CMD regions. In [40], these regions are large and strategically chosen to sample stars of different ages or specific stellar evolutionary phases and to take into account uncertainties in the stellar models: this solution guarantees an optimal statistics, but has the drawback of underexploiting the fine structure of the CMD. Another possibility is to choose a fine grid of regions (see, e.g., $[51,52]$ ), counting how many predicted and observed stars fall in each region: the temporal resolution is higher, but the Poisson noise is the new drawback. An intermediate solution is to build a variable grid, coarser where the density of stars is lower and finer when the density is higher (see, e.g., $[45,53]$ ). At this level, ad-hoc weighting of some regions can be introduced both to emphasize CMD regions of particular significance for the determination of age, and to mask those regions where stellar evolutionary theory is not robust.

Other authors avoid to grid the CMD: for instance, in [38] each model point (apparent magnitude and colour) is replaced with a box with a Gaussian distributed probability density (the photometric error). The total likelihood of a model is the product of the probabilities of observing the data in each box. The idea of this method is equivalent to use blurred isochrones (each point is weighted by the Gaussian spread), so that the photometric uncertainty is embodied in the theoretical model.

3.3. Maximum Likelihood. The next step is to choose a criterion for the comparison between synthetic and observational CMDs. For any grid scheme, once binned, data and synthetic CMDs are converted in colour-magnitude histograms. So, the new problem is to quantify the similarity among $2 \mathrm{D}$ histograms. One possibility is to minimize a $\chi^{2}$ likelihood function: when the residuals (differences among theoretical and observed star-counts in the CMD regions) are normally distributed, all models that have a $\chi^{2}$ greater than the best fit plus one are rejected. However, when the distribution is not normal, a $\chi^{2}$ minimization leads to a wrong solution. This motivates the use of the Poisson likelihood function instead of the least-squares fit-to-data function. In order to determine the uncertainties around the best model, a valid alternative is to use a bootstrap test: the original data are randomly resampled with replacements to produce pseudoreplicated datasets. This mimics the observational process: if the observational data are representative of the underlying distribution, the data produced with replacements are copies of the original one with local crowding or sparseness. The star formation recovery algorithm is performed on each of these replicated datasets. The result will be a set of "best" parameters. The confidence interval is then the interval that contains a defined percentage of this parameter distribution.

One aspect deserves closer inspection: the minimization of a merit function of residuals ( $\chi^{2}$ or Poisson likelihood) is a global measure of the fit quality. The first side effect is purely statistical: low-density regions of the CMD may be ignored in the extremization process, whereas well-populated phases (as the MS) are usually well reproduced. This is a problem of contrast: low-density regions are Poisson dominated, thus, they are much easier to match with respect to the well populated regions.

A Monte Carlo method can be used with great success to evaluate this bias: building synthetic CMDs from the best set of parameters and re-recovering the SFH can allow to remark any statistical discrepancy. Then, a straightforward solution is to enhance the significance of the discrepant regions of the CMD (with appropriate weights).

Another problem is connected with the theoretical ingredients we have used in the models: first of all, stellar evolution models are not perfect and computations by different groups show systematic differences (see, e.g., for a review [54]). Model atmospheres are often unreliable for cool and metal rich stars. Moreover, our models are only covering a part of the possible parameter space and some degree of freedom 
(additional metallicities, mass loss, overshooting, etc.) may have been neglected. In this case, our best model is only the best (in a relative sense) of the explored parameter space, not necessarily a good one.

In order to deal with these undesirable effects, the residuals can be placed in the CMD, identifying all the regions where the discrepancy between observed and predicted starcounts is larger. If the residuals are larger and concentrated in some part of the CMD, we may understand what is the reason for the discrepancy and take it into account. For instance, a poor fit in the red giant branch, less populated than the main sequence but morphologically well defined in colour (and for this reason often neglected in $\chi^{2}$ minimization), may suggest a wrong metallicity, a different mixing length parameter, or a wrong colour transformations.

3.4. Wondering in the Parameter Space. The main drawback of Maximum Likelihood (ML) approaches is the computational burden. Algorithms that find the ML score must search through a multidimensional space of parameters, using, for instance, derivative methods, like Powell's routine, or nonderivative ones, like the downhill simplex routine, or genetic approaches (see, e.g., [53]).

These techniques are not guaranteed to find the peak, but work relatively well for a limited number of parameters. Traditionally, this question has been tackled by constructing synthetic CMDs from an SFH built as a series of contiguous bursts and finding the amplitudes of each burst that give the maximum probability to have produced the data: the synthetic CMD is now a linear sum of the partial CMDs produced from a single realization for each burst. In this way, a huge parameter space can be explored: rather than calculating a complete CMD for each SFR(t), the partial CMDs can be linearly combined to build a CMD for any SFR(t). In order to reduce the Poisson noise, the partial CMDs are simulated with many more stars than observed (typically, 100 times more).

The computer time spent for building the final CMD is only that needed to go through a finite number of models, simply equal to the number of combinations of $Z(t)$ relations, IMF slopes, reddenings, and distance moduli times the number of age bins in the solution.

Age Bins: disentangling a stellar population showing both very recent (Myr) and very old (Gyr) episodes of star formation is not straightforward. Only low-mass stars survive from ancient episodes because their evolutionary timescales are very long: small CMD displacements, for example, due to photometric errors, can bias their age estimates up to some Gyr. In this case, increasing the time resolution, besides being time-consuming, may produce unrealistic star formation rates due to misinterpretations. Hence, the choice of temporal resolution must follow both the time scale of the underlying stellar populations and the data scatter (photometric errors, incompleteness, etc.). A practical way out is to use a coarser temporal resolution for the older epochs, which (1) allows us to avoid SFH artifacts at early epochs, (2) reduces the Poisson noise, and (3) reduces the parameter space. Figure 5 shows a possible time stepping (see, [27]). Finally, it is worth noting that the choice of each set of age bins will prevent to identify any SF episode shorter than the bin duration: for instance, the $1 \mathrm{Gyr}$ lull (between 2 and $3 \mathrm{Gyr}$ ago) in the star formation history, as simulated in the Figure 1(c), will result in a lower (half) activity in the 7th age bin (1-3 Gyr).

In the following sections we describe some numerical experiments illustrating the reliability of a typical ML algorithm. In particular, the sensitivity of such algorithm to several physical uncertainties is outlined. These examples expand the discussions and results by [46]. The experiments described here are based on different set of tracks, mass spectrum, and photometric errors/completeness, but the results are the same as in [46], thus showing that they are independent of these assumptions. Other instructive examples can be found in $[40,54,55]$.

3.5. A Practical Example. To describe how an ML procedure works, let us build a fake galaxy assuming for the sake of simplicity a constant star formation rate between now and $13 \mathrm{Gyr}$ ago and a metallicity fixed at $Z=0.004$. We put it at the distance of the closest dwarf irregular galaxy, the Small Magellanic Cloud (SMC), $(m-M)_{0}=$ 18.9, and adopt the SMC mean foreground reddening $E(B-V)=0.08$. To minimize statistical fluctuations, the Monte Carlo extractions are iterated until we have 30000 stars brighter than $V=23$, which roughly corresponds to about 100000 stars in the entire CMD. Photometric errors and incompleteness as obtained in actual HST/ACS SMC campaigns [27] are convolved with the synthetic data, producing a realistic artificial population. This fake galaxy will be used as a reference set in all the following exercises.

To recover its SFH, we have gridded the CMD in small bins of colour and magnitude (0.1 mags large) and we have minimized a Poisson likelihood. The time stepping for the partial CMDs is as follows (going backward in time from the present epoch to $13 \mathrm{Gyr}$ ago): $100 \mathrm{Myr}, 400 \mathrm{Myr}, 500 \mathrm{Myr}$, 1 Gyr, 2 Gyr, 3 Gyr, 3 Gyr, 3 Gyr. A bootstrap technique is implemented to determine the final uncertainties.

Figure 6(a) shows the CMD of our reference fake galaxy, and Figure 6(b) the SFH recovered from it, using only stars brighter than $V=23$ (i.e., $M_{V}=3.85$ ), for a selfconsistency check. As expected, the retrieved SFR is fitted by a constant value. We will use this basic experiment as a starting point for a series of exercises aimed at describing the major uncertainties affecting the synthetic CMD method.

\subsection{Uncertainties Affecting the Synthetic CMD Procedures.} Contrary to real cases, in the reference case of Figure 6 we have all the information: all parameters are known and the data are complete down to $23\left(M_{V}=3.85\right)$. Real galaxies are far from this ideal condition. Inadequate information or uncertainty about the assumed parameters can influence the identification of the best SFH. The major sources of uncertainty are primarily the IMF, the binary fraction, and the chemical composition. From the observational point of view, the completeness level is another important factor. Moreover, population synthesis methods make a number of simplifications to reduce significantly the computational load; for example, reddening constant across the data, same 


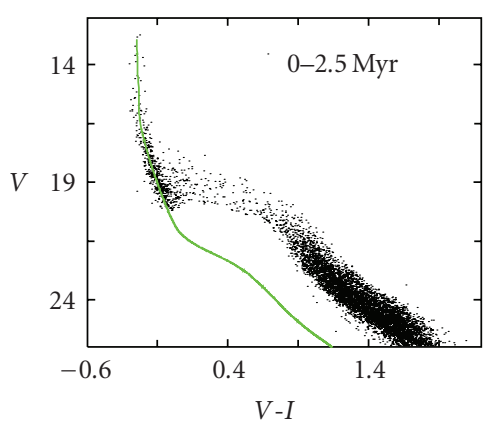

(a)

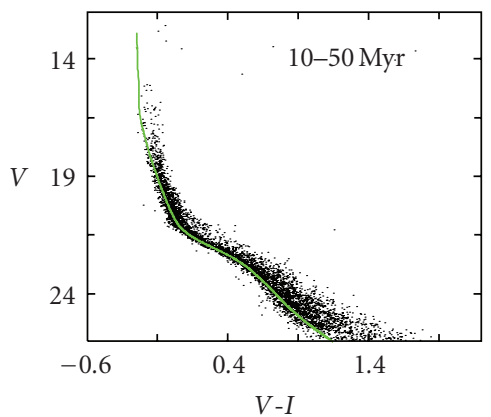

(d)

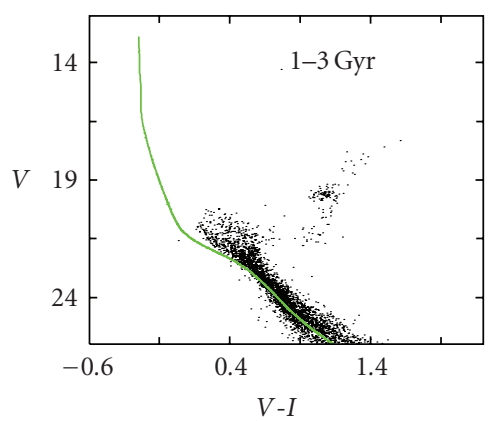

(g)

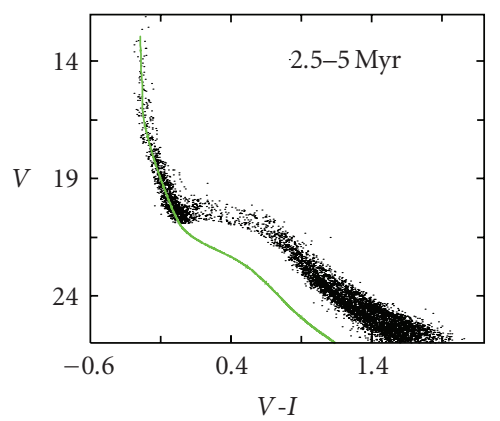

(b)

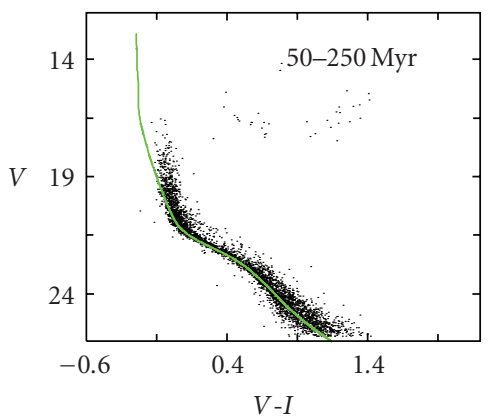

(e)

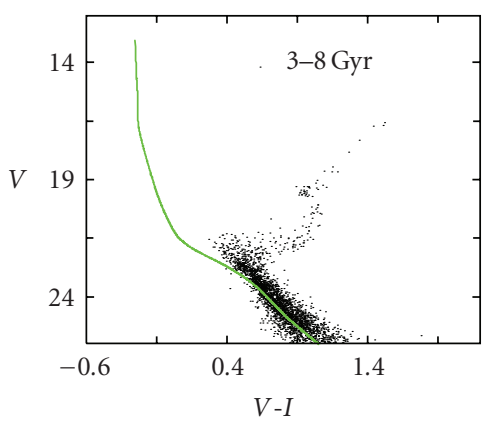

(h)

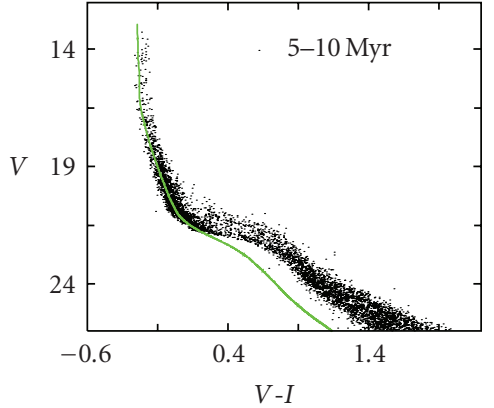

(c)

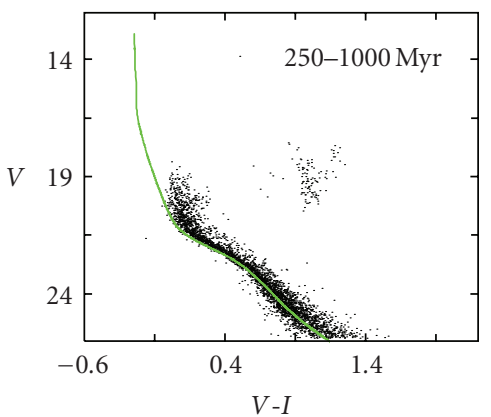

(f)

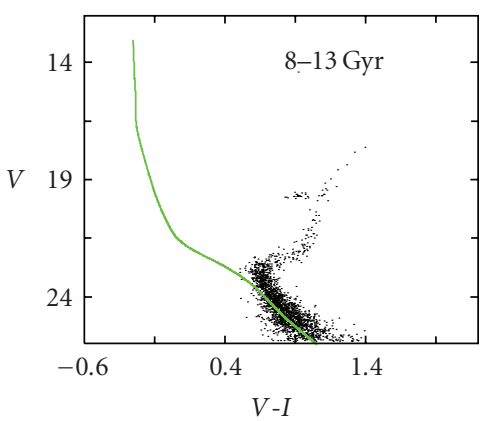

(i)

FIgURE 5: Partial theoretical CMDs. Each one is generated with a constant star formation rate, for the age interval pointed out in the label. The bright part of the main sequence is dominated by high-mass stars so the CMD time step has to be shorter.

distance for all stars, linear age-metallicity relation, and so forth. Dropping these simplifying assumptions considerably complicates all analyses of the CMD properties.

There are two main strategies to face the complexity of the problem. One is to increase the number of free parameters in the model. For example, the work in [56] recovers simultaneously distance, enrichment history, and SFR of the local dwarf LGS 3 . The other is to reduce the data complexity by means of additional information; for instance, the metallicity may be estimated from appropriate spectroscopy and multiband observations may help to disentangle the reddening.

In the following subsections we test the reliability of the star formation recovery when the uncertainties related to each parameter are taken into account individually. A word of caution is necessary for the interpretation of these exercises: in each case, we show how the recovery of the SFH of the reference fake galaxy is affected by forcing the procedure to adopt a specific (and in most cases wrong) value for the tested parameter. This is aimed at emphasizing the effect of that parameter. In the derivations of the SFH of real galaxies, the parameter values are all unknown (which complicates the derivation), but the selection procedure is allowed to cover all the meaningful ranges of values and can therefore distinguish which combinations allow to maximize the agreement with the data.

3.7. Completeness. The numerical experiment seen in Section 3.5 represents an ideal situation: (1) the SMC is one of the closest galaxies, (2) HST/ACS currently provides the top level photometry in terms of spatial resolution and depth. At the distance of the SMC $(60 \mathrm{kpc})$, ACS photometry can be $100 \%$ complete to $V \approx 24-25$. Farther galaxies and/or images from ground-based telescopes have larger photometric errors and more severe incompleteness. For comparison, the optical survey conducted at the Las 


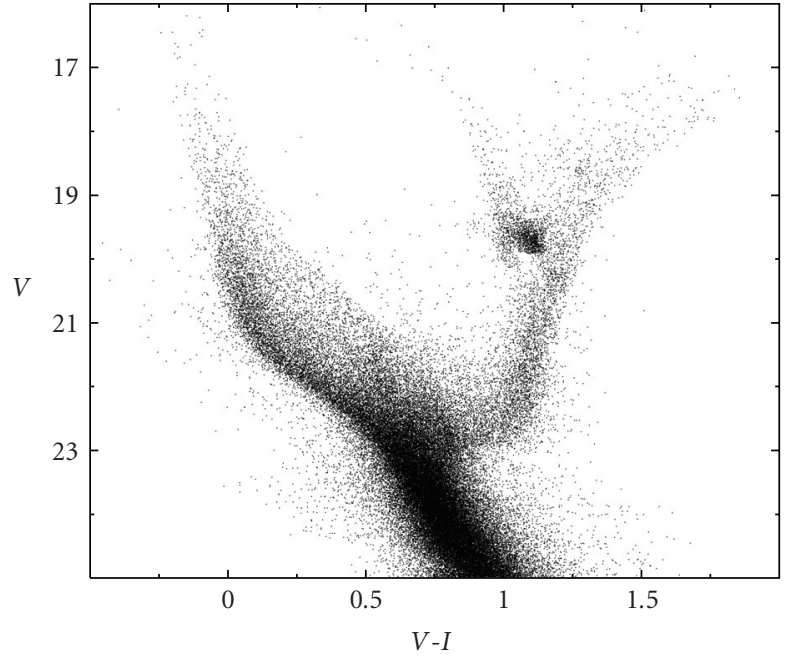

(a)

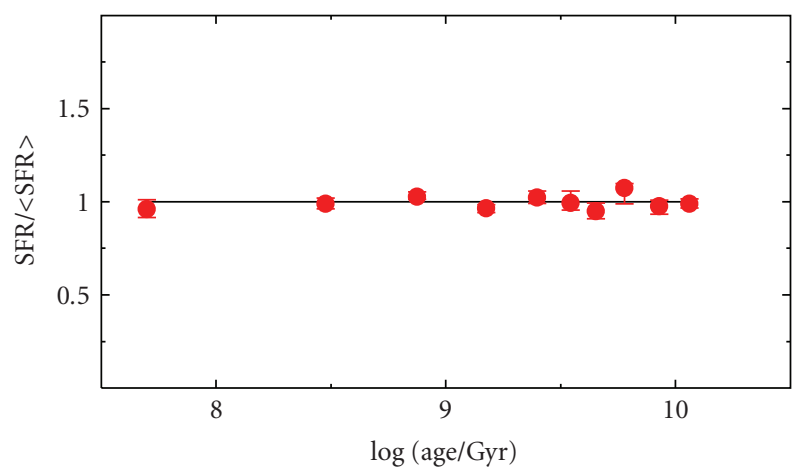

(b)

FIgURE 6: Starting case for the experiments on the uncertainties on the retrieved SFH due to different factors. In (a) we show an artificial population of stars generated with constant SFR, Salpeter IMF, constant metallicity $Z=0.004$ [23], $(m-M)_{0}=18.9$, $E(B-V)=0.08$, and the HST/ACS errors and completeness of the photometry in the SMC field NGC602 [27]. In (b) the input (solid line) and the recovered (dotted) SFH are compared.

Campanas Observatory $1 \mathrm{mt}$ Swope Telescope, to trace the bright stellar population in the Magellanic Clouds, is 50\% complete to $V \sim 21-22$ (see [57]).

To demonstrate the importance of the completeness limit, we perform the star formation recovery using only stars brighter than $V=21$ and $V=22$. The results are displayed in Figure 7. It is evident that the deeper the CMD, the higher the chance to properly derive the old star formation activity. Compared to $V=23$ case, where the SFR recovery is accurate and precise at any age, the quality already drops when the completeness limit is at $V=22$ : the larger error bars at old epochs reflect the fact that the only signature of the oldest activity comes from evolved stars, less frequent, and much more packed in the CMD than the corresponding MS stars. Rising the limiting magnitude at $V=21$ further worsens the result, and the recovered SFH is a factor of 2 uncertain for ages older than 1-2 Gyr.
These results are actually optimistic: we have analyzed different levels of completeness with the same photometric errors (HST/ACS), but this is an utopic situation. More distant galaxies have a less favorable completeness limit, and also the photometric error is larger. In these cases, an additional blurring occurs.

3.8. IMF. A large body of evidence seems to indicate that (1) the stellar IMF has a rather universal slope, (2) above $1 M_{\odot}$ the IMF is well approximated by a power law with Salpeterlike exponent [58], (3) below $1 M_{\odot}$ the IMF flattens.

According to [28], the average IMF (as derived from local Milky Way star-counts and $\mathrm{OB}$ associations) is a three-part power law, with exponent $\alpha=2.7 \pm 0.7$ for $m>1 M_{\odot}$, $\alpha=2.3 \pm 0.3$ for $0.5 M_{\odot}<m<1 M_{\odot}, \alpha=1.3 \pm 0.5$ for $0.08 M_{\odot}<m<0.5 M_{\odot}$. Other authors, in the past, have proposed different (although somewhat similar) slopes for the IMF of various stellar mass ranges (e.g., [29, 59, 60]). Given these uncertainties, it is necessary to evaluate how this impacts the possibility to infer the SFH. In fact, we have the degeneracy condition that false combinations of IMF and SFH can match as well the present day mass function (the current distribution of stellar masses) of MS stars. To quantify it, three fake populations were generated with different IMF exponents ( $\alpha=2,2.35$ and 2.7), but the SFH searched using always 2.35. The results are shown in Figure 8.

To interpret these results, one must recall that recent steps of star formation are still populated by the entire mass spectrum, while old steps see only low-mass stars because the more massive stars born at those epochs have already died. For old stars, a steeper IMF is almost indiscernible from a more intense star formation. In fact, even if evolved stars are included in the SFH derivation, the mass difference between a star at the RGB tip and those at the MS turn-off is only few hundredths of solar masses: too small for allowing the identification of any IMF effect.

For young stars the situation in different. Any attempt to reproduce with an IMF steeper than that of the reference population the number of stars on the lower MS requires a stronger SF activity, but this (wrong) solution leads to overestimating the number of massive stars. Hence, for young stars, IMF and SFH are not degenerate. However, an automatic optimization algorithm, if not allowed to search better solutions including also the IMF among the free parameters, inevitably faces the impossibility to accommodate the number of both massive and low-mass stars, by choosing a compromising recent SFH giving higher weight to the more populated (although less reliable) CMD region.

As shown in Figure 8 the automatic fit tends to overestimate the age of any population whose IMF is actually steeper than the adopted one, and vice versa, for a flatter IMF. Again, we remark that the automatic solution is only the best solution in a parameter space where the IMF is fixed, not necessarily a good one: if the CMD of the recovered $\mathrm{SFH}$ is compared with the reference CMD, we immediately recognize that the ratio between low and massive stars is wrong. In other words, to figure out whether our "best" 


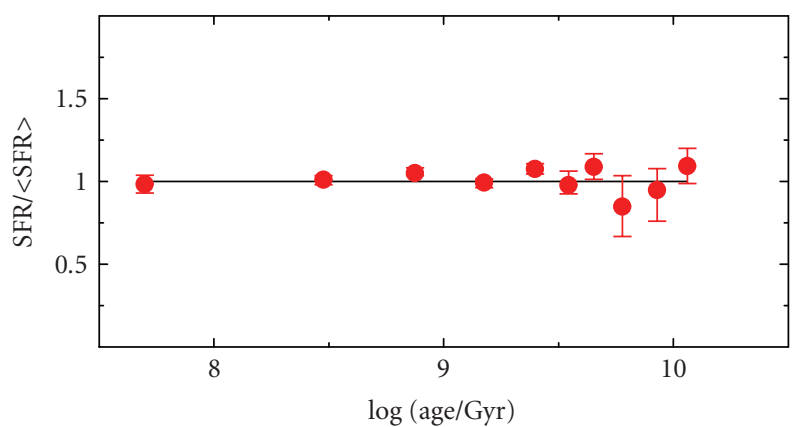

(a)

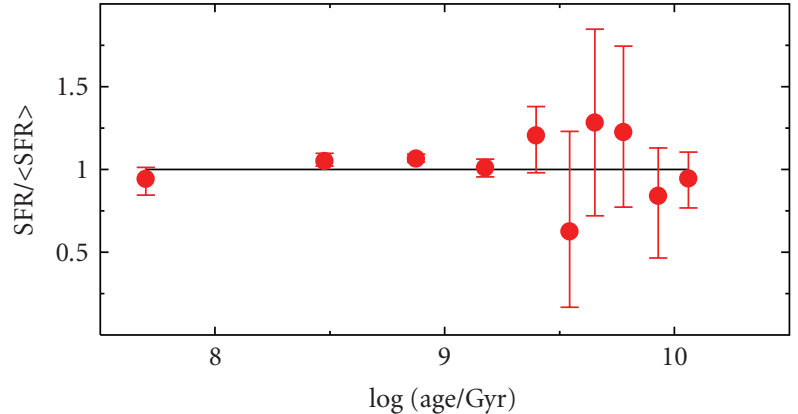

(b)

FIGURE 7: Effect of different completeness limits: in Figure 7(a) the SFH is recovered using only stars brighter than $V=22$. In this case, most of the information is still retrieved. In (b) this limit is lowered at $V=21$ as expected, most of the old star formation (older than 1 Gyr) is much more uncertain.

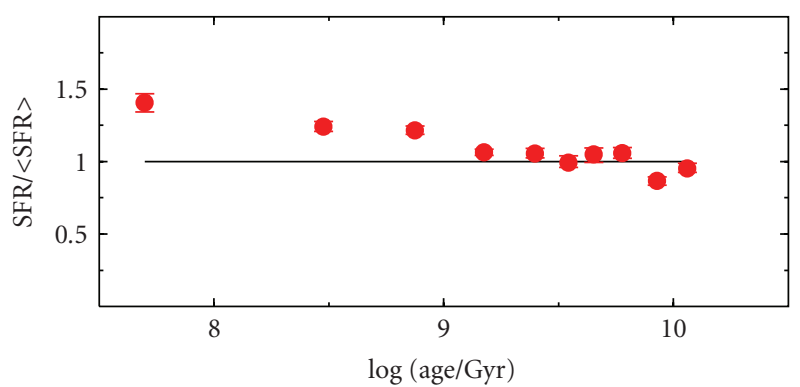

(a)

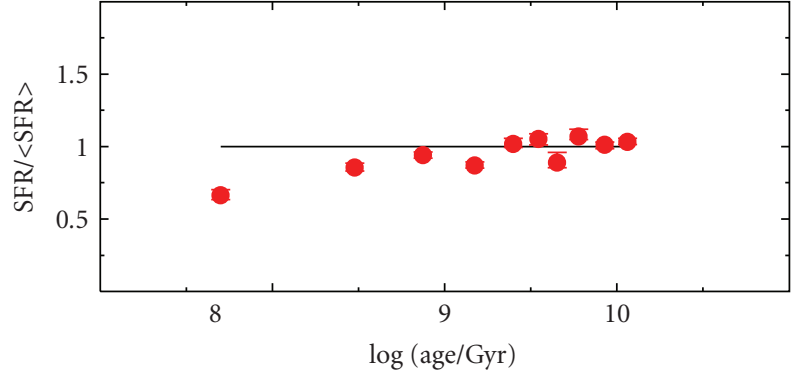

(b)

FIGURE 8: Effect of different IMFs. SFH recovered assuming Salpeter's IMF exponent (2.35) for synthetic stellar populations actually generated adopting different IMF (labeled in each panel) and a constant SFR.

solution is actually acceptable, it is always crucial to compare all its CMD results with the observed one.

3.9. Binaries. Another source of uncertainty is the percentage of stars in unresolved binary systems and the relative mass ratio. The presence of a given percentage of not resolved binary systems affects the CMD morphology. The aim, here, is to see if these effects can destroy or alter the recovered information on the SFH. In order to perform this analysis, we build fake populations using different prescriptions for the binary population $(10 \%, 20 \%$, and $30 \%$ of binaries with random mass ratio), but the SFH is searched ignoring any binary population (i.e., assuming only single stars). Our models do not include binary evolution with mass exchange, thus we assume that each star in a double system evolves as a single star.

Figure 9 shows the results, as for the IMF, also in this case a modest systematic effect is visible. This is because the stars in binary systems are brighter and redder than the average single star population (for an in depth analysis see, e.g., [61]). For the recent SFH, this corresponds to moving lower MS stars from a star formation step to the contiguous older step: in this way, the most recent star formation step is emptied of stars, mimicking a lower activity. Intermediate SF epochs are progressively less affected, because some stars get in and some stars get out of the step bin. For the oldest epochs, the situation is opposite: the binary effect is to move stars toward younger bins. Here, the SGB, that is, the main signature of any old population, is brighter because of the binaries, mimicking a younger system.

3.10. Metallicity and Metallicity Spread. The precise position of a star on the CMD depends on the chemical composition, namely, the mass fraction of hydrogen, helium, and metals ( $X, Y, Z$, respectively). The $Z$ content mainly changes the radiative opacity and the $\mathrm{CNO}$ burning efficiency: the result of a decreasing $Z$ is to increase the surface temperature and the luminosity of the stars. This has two consequences of relevance for us: (a) metal poor stars have a shorter lifetime compared to the metal-rich ones (because overluminous and hotter), (b) a metal-poorer stellar population is bluer but can be mistaken for a younger but metal-richer population.

To test these effects, the first stars (ages older than 5 Gyr) in our reference fake population are built with a slightly different metallicity $(Z=0.002)$ than the younger objects, which have the usual $Z=0.004$. Then, we recover the SFH by adopting a model with $Z=0.004$ independently of age. The results are shown in Figure 10: neglecting that the oldest population of our galaxy was slightly metal poorer, systematic, nonnegligible discrepancies appear in the recovered SFH. It is the classical age-metallicity degeneracy: to match the blue-shifted sequences of old metal poorer stars, 


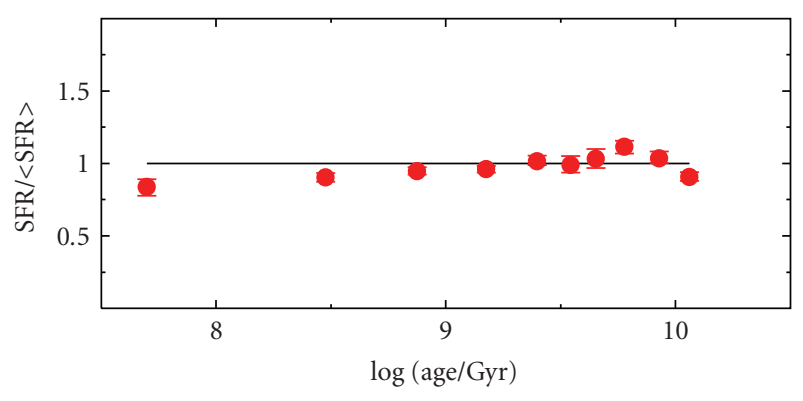

(a)

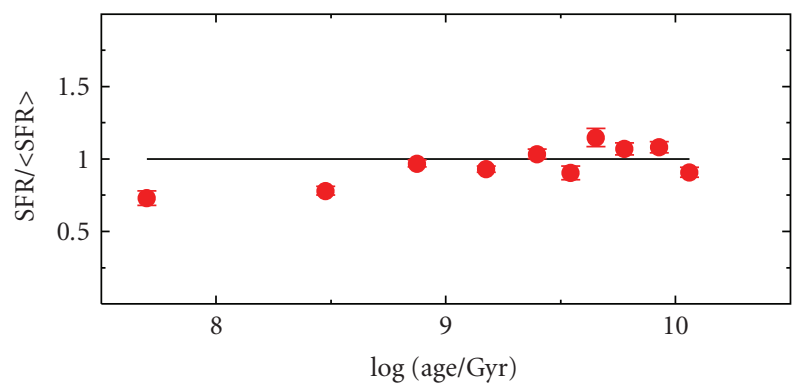

(b)

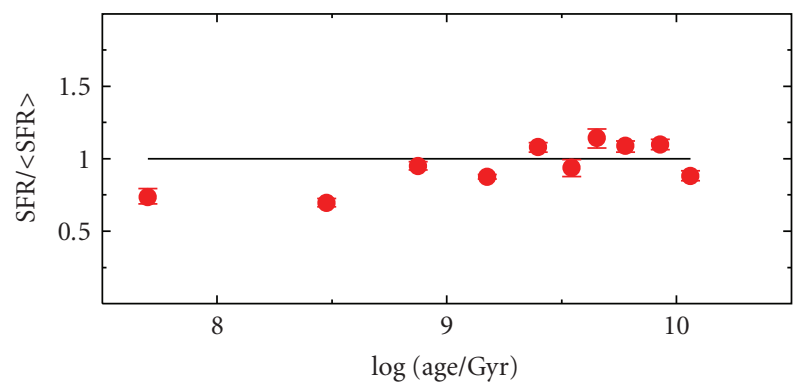

(c)

FIGURE 9: Effect of unresolved binaries. Three fake populations are built with different percentage of binary stars (10\%, 20\%, and 30\%). The SFR is recovered using only single stars.

our models with wrong metallicity must be younger. Note that the overall trend of the young SFR is not significantly biased, while the old SFR is now significantly different.

This result is a strong warning against any blind attempt to match the CMD with a single (average) metallicity, especially considering that many galaxies exhibit a pronounced age metallicity relation.

During and at the end of their life, stars pollute the surrounding medium, so we expect that more recently formed stars have higher metallicity and helium abundance than those formed at earlier epochs. The progressive chemical enrichment with time results from the combined contribution of stellar yields, gas infall and outflows, mixing among different regions of a galaxy. Observational studies have shown that several galaxies reveal a metallicity spread at each given age.

In [46], the SFH sensitivity to a metallicity dispersion is tested: several fake populations were generated with a mean metallicity $Z=0.02$ plus a variable dispersion from

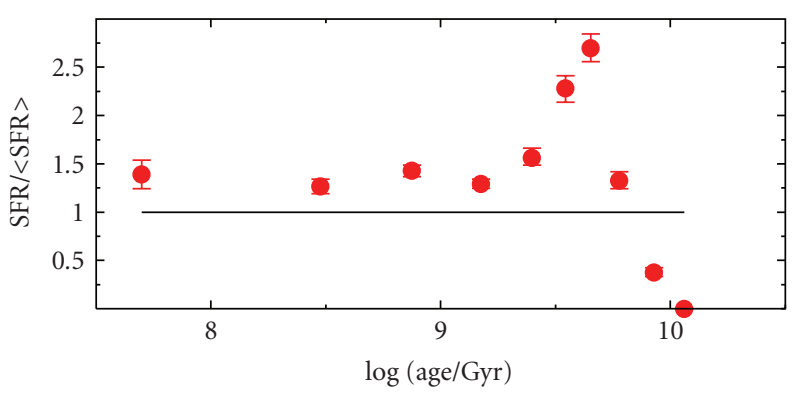

FIGURE 10: Sensitivity test to metallicity. The reference fake galaxy has a variable composition: $Z=0.004$ for stars younger than $5 \mathrm{Gyr}, Z=0.002$ for older stars. The red dots represent the SFR as recovered using a single metallicity $Z=0.004$ for all epochs.

$\sigma=0.01 \mathrm{dex}$ to $\sigma=0.2 \mathrm{dex}$ in $[\mathrm{Fe} / \mathrm{H}]$. Then, the SFH was searched adopting in the model the same mean metallicity of the artificial data, but without metallicity spread. The results are shown in Figure 11. As described earlier, $\sigma=0.1 \mathrm{dex}$, the retrieved SFH differs significantly from the true one: this numerical experiment points out that the metallicity dispersion can be a non negligible factor.

3.11. Helium Content. The SFH retrieval must be also tested against possible variations of the helium abundance for a given metallicity. The abundance of $\mathrm{He}$ and metallicity influence the stellar structure through the molecular weight. Increasing $Y$ corresponds to increasing the molecular weight and affects the hydrostatic equilibrium. The pressure decreases and the star shrinks (producing heat), reaching a new equilibrium characterized by a smaller radius and a higher central temperature. As a consequence, the efficiency of the central burning increases and this makes the star brighter and hotter.

Helium absorption lines appear only in the spectra of very hot stars. Hence, the traditional procedure to infer the helium abundance of a standard stellar population cannot be via direct measures and is instead based on the correlation (which is assumed linear) between the helium mass fraction $Y$ and the metal abundance $Z$.

In order to explore the effects of a wrong choice of the helium content, we have built a reference fake population with $Z=0.004$ and $Y=0.27$ and we have tried to recover its SFH using a $Z=0.004$ model coupled with $Y=0.23$, which is obviously a very extreme assumption and, therefore, provides a stringent upper limit to the possible $Y$ effects on the SFH. The result is shown in Figure 12: there are a number of small variations, but the general morphology is well reproduced. The small features of the recovered SFR are blended (the peaks are attenuated) but the overall trend is still recovered.

3.12. Reddening and Distance. In order to test how wrong choices of reddening and distance invalidate the possibility to recover the right $\mathrm{SFH}$, we consider two extreme situations: first, the fake galaxy is put at $(m-M)_{0}=18.6$, with the models still assuming $(m-M)_{0}=18.9$ second, the fake 


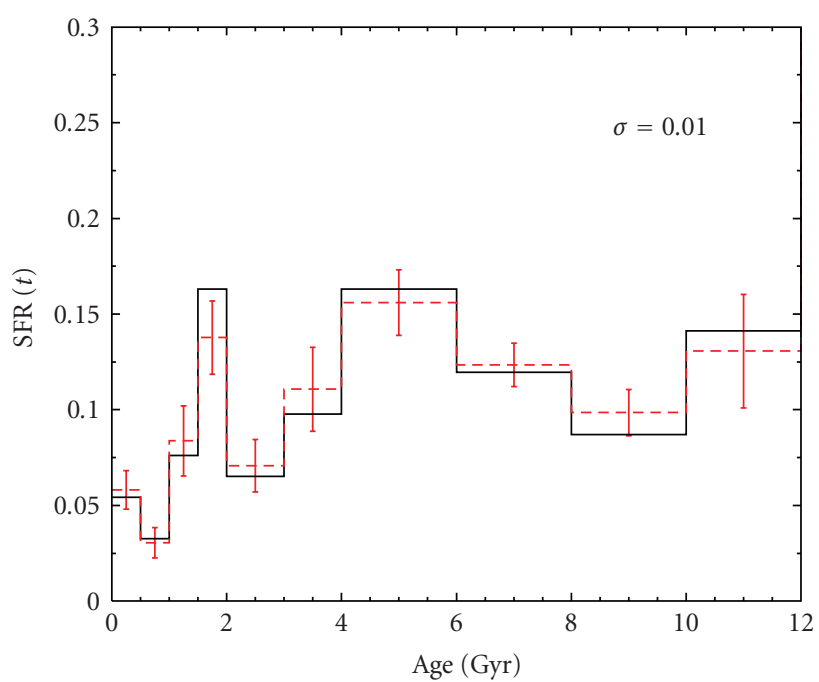

(a)

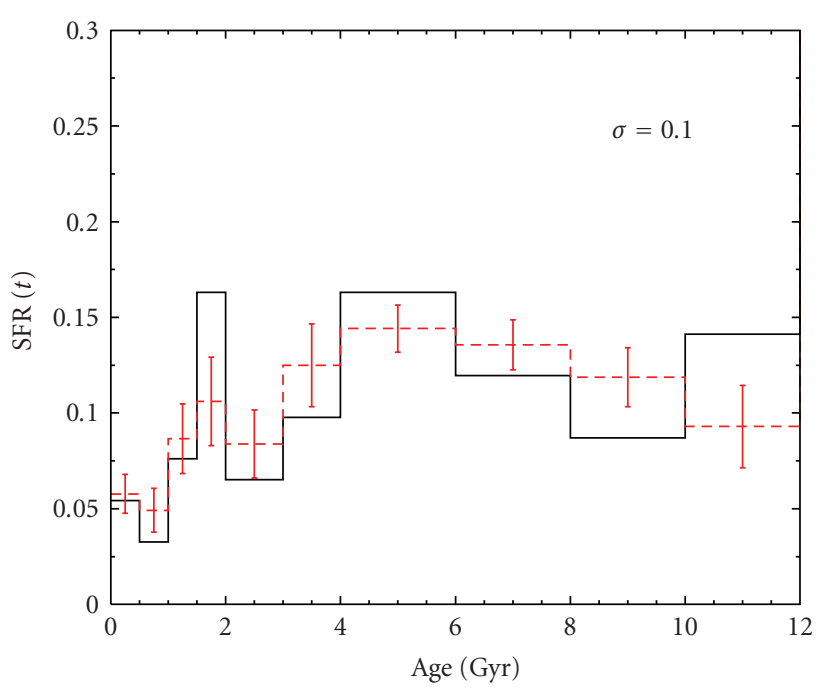

(c)

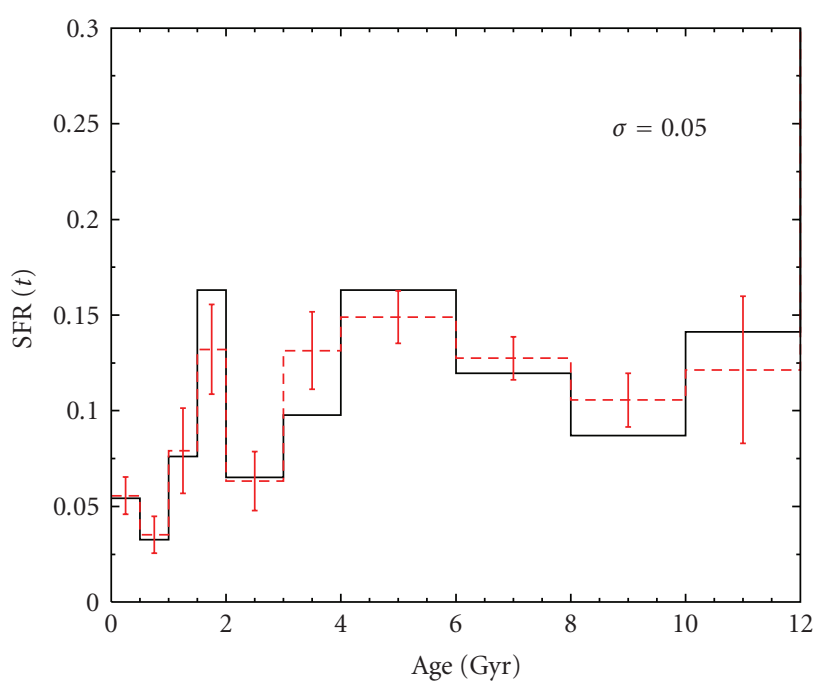

(b)

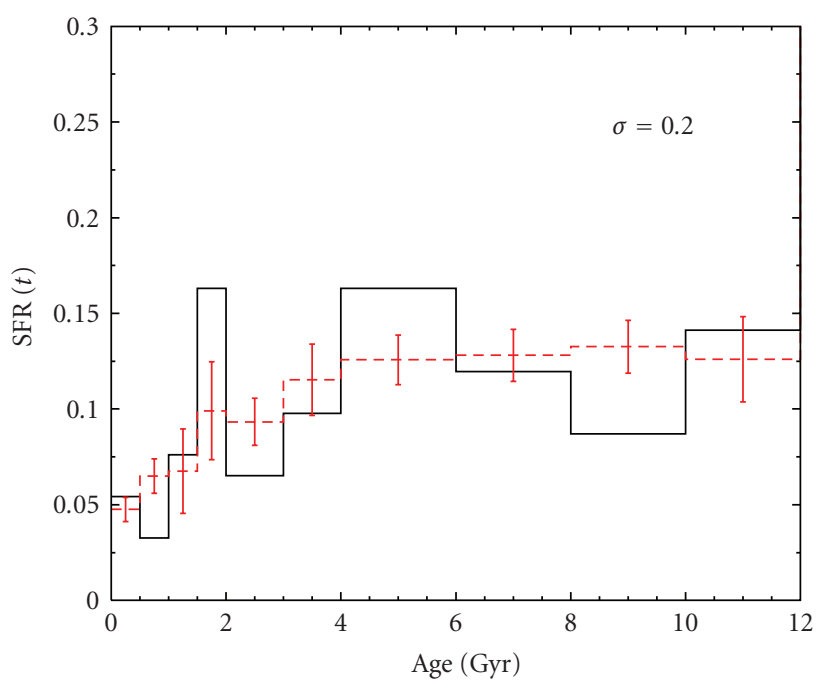

(d)

FIgURE 11: Sensitivity test to the metallicity dispersion. Solid line: SFH assumed for the fake population. Dashed line: recovered SFH. The $\sigma$ value indicates the dispersion in $[\mathrm{Fe} / \mathrm{H}]$, used for the reference artificial data. The model has the same mean metallicity, but no dispersion.

galaxy is reddened using $E(B-V)=0.16$ mag, while the models adopt $E(B-V)=0.08$. The results are shown in Figure 13: it is evident that the SFH is not recovered, and shows large deviations from the reference case at any epoch. However, it is worth to remind that we have derived the best models from a blind $\chi^{2}$ minimization: it is clear that at the distance of SMC, with the beautiful HST photometry available, both a visual inspection of the CMD morphology (in particular the blue MS envelope) and the evaluation of the $\chi^{2}$ probability would be effective to reject all the models with wrong distance and reddening. The problem is more challenging for more distant galaxies, where the only observables are massive stars and reddening and distance are difficult to constrain.

Other potential problems are related to differential reddening and line-of-sight depth. There are several indications that some galaxies or portions of them are affected by differential reddening. For instance, young stars can be still surrounded by relics of their birthing cocoon material and suffer an additional amount of absorption. The first signature of a differential reddening is the red clump morphology when it appears elongated and/or tilted (see, e.g., [62]). Another effect is a smeared appearance of colour and magnitude in any stellar evolution sequence of the CMD.

A finite line-of-sight depth is a natural expectation, at least for nearby galaxies where the physical extension can be a nonnegligible fraction of their distance from us. As an example, according to [63], the SMC may have a depth of 14-17 kpc, corresponding to a spread in magnitude of few tenths of magnitude. This effect may alter the evolutionary information from the clump and the RGB. A correct description of the stellar populations thus requires analyses 


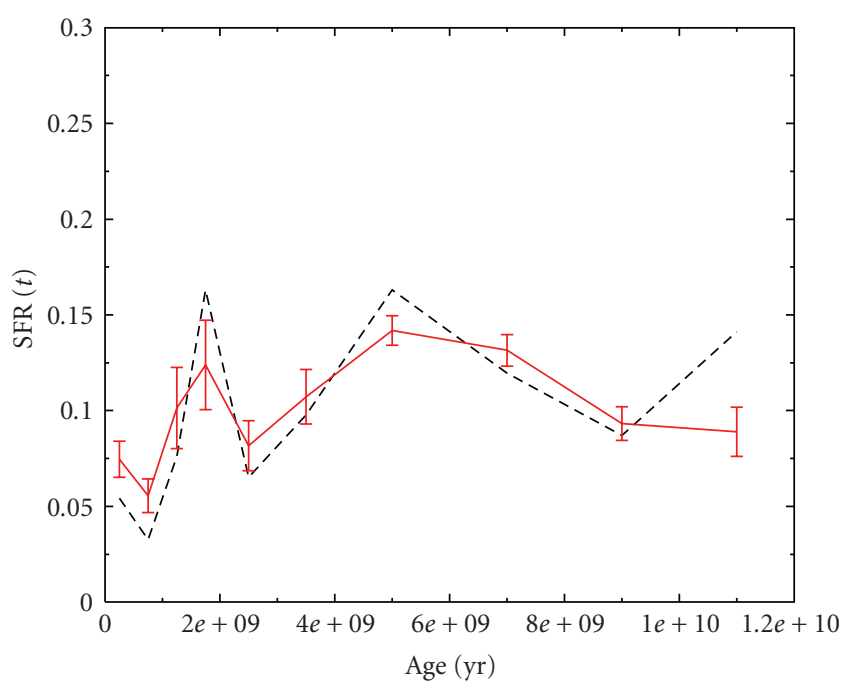

FIGURE 12: Experiment of the helium effect on the SFH: reference fake population and model have the same metallicity $Z=0.004$, but different helium content $(Y=0.23$ for the model and $Y=0.27$ for the artificial data). The black dashed line is the input SFH. The red solid line is the recovered SFH.

involving the spatial structure. Both differential reddening and line-of-sight effects can be dealt with as additional free parameters.

In order to check how the recovered SFH is influenced by differential reddening and line-of-sight spread, we built two fake populations with the following features: the first has a reddening dispersion $E(B-V)=0.08 \pm 0.04$, the second has a distance spread $(m-M)_{0}=18.9 \pm 0.2$. Figure 14 shows the result when the $\mathrm{SFH}$ is searched using the canonical combination $E(B-V)=0.08$ and $(m-M)_{0}=18.9$. The reference SFH is still fully recovered. The reason is in the random nature of these uncertainties that produce a blurred CMD, but not a systematic trend.

3.13. Additional Issues. The previous experiments do not exhaust all the possible sources of uncertainty. Rather, they represent the best understood and manageable ones: among others, convection, atmospheres, and colour transformations, stellar rotation, mass exchange in binary systems may be relevant as well. Moreover, we have explored each single bias separately, while in a real galaxy, several uncertainties may be at work simultaneously with different intensities. In this case, the final effect may not be the simple summation of the previous results: some of the effects may compensate each other or conspire to build an uncertainty larger than the sum of the individual uncertainties.

For example, in the metallicity experiment, when the reference old population was metal poorer than in the search procedure, the retrieved SFH resulted younger. However, this is true only if the best SFH is searched with fixed reddening. Letting the reddening vary could lead to a different SFH (and reddening).

To summarize, the final uncertainty on the recovered SFH strongly depends on which parameter space is explored.
Generally speaking, when the best photometric conditions are achieved and all the parameter space is properly covered, within the reached lookback time the error on the epochs of the SF activities is around ten percent of their age and that on the SFR is of the order of a few. With poorer photometry or with coarser procedures the uncertainties obviously increase, but the qualitative scenario is usually reliably derived.

\section{Star Formation Histories of Resolved Dwarfs from Synthetic CMD Analyses: Results}

In spite of the uncertainties described earlier in the identification of the best solution for the SFH, the synthetic CMD method is extremely powerful in reducing the range of acceptable scenarios, that is, the range of values of the various parameters. As demonstrated every time different synthetic CMD procedures have been applied to the same galaxy region, all the solutions come out consistent with each other (see, e.g., the Coimbra experiment on the LMC bar [50], and IC1613 [64]). We can, therefore, dare drawing some general conclusions from the results obtained so far with this method.

Since the dawn of its application, the method immediately proved its power. First, it was found that the SFH differs significantly from one galactic region to the other even in tiny systems such as WLM, the first dwarf irregular (dIrr) in the Local Group to which the method was applied [30]. As soon as a few other nearby irregulars were studied, it turned out that, contrary to the common belief of the time, the SF activity in late-type dwarfs within the lookback time spanned by the available photometry has occurred in long episodes of moderate intensity, separated by short quiescent phases, rather than in short episodes of strong intensity, separated by long quiescent intervals $[31,33-35,66]$. In other words, a gasping [34] rather than a bursting regime.

Nowadays, one can resolve individual faint/old stars in galaxies of the LG and its immediate vicinities, and infer their SFHs over long lookback times. In the (still few) cases when the oldest MSTO is reached, the SFH can be derived over the entire Hubble time, as already achieved in some regions of the LMC $[43,62,67-70]$, of the SMC $[27,71,72]$ and in Leo A [65]. As an example, Figure 15 shows the CMD obtained by [65] from ACS imaging of the dIrr Leo A, located at 800 $\mathrm{kpc}$ from us [52], and the resulting SFH. In Leo A the star formation activity was present, although quite low at the earliest epochs, and $90 \%$ of the activity occurred in the last $8 \mathrm{Gyr}$, with the main peak around 2 Gyr ago and a secondary peak a few hundreds Myr ago. This SFH is very similar to that of fields in the LMC, SMC, IC1613 [73], and other latetype galaxies and we can consider it typical of dIrrs: a rather continuous star formation since the earliest epochs, but with significant peaks and gasps. Notice that the main SFR peak in dIrrs rarely occurs at the most recent epochs.

The high spatial resolution of HST cameras also allows to spatially resolve the SF activity, at least within relatively recent epochs. For instance, the work in [74, 75] has measured the SF activity over the last $0.5 \mathrm{Gyr}$ in all the subregions of the dIrrs Gr8 and Sextans A, close to the 


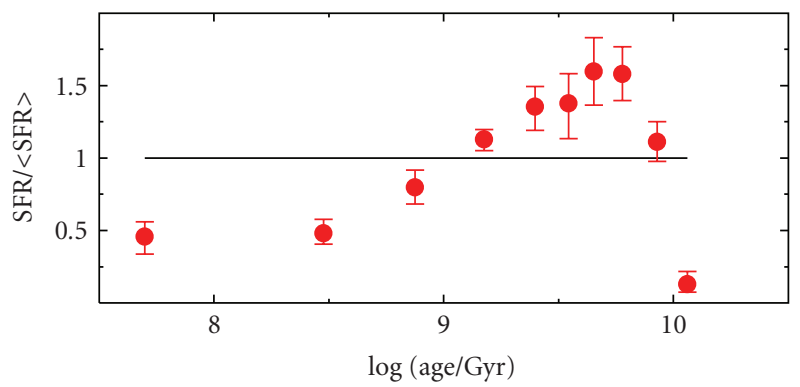

(a)

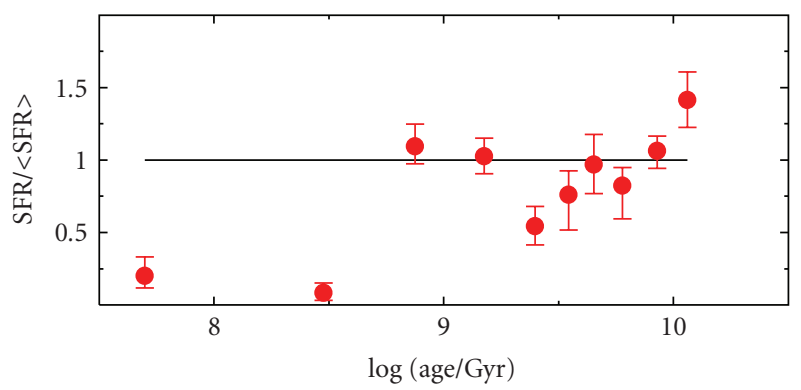

(b)

FIGURE 13: Sensitivity test to reddening and distance. Red dots represent the recovered SFH. In (a), the reference fake population is built with a distance spread $(m-M)_{0}=18.6$, while the model used to retrieve the SFH adopts $(m-M)_{0}=18.9$. In (b),the reference fake population is built with reddening $E(B-V)=0.16$, while the model used to retrieve the SFH adopts reddening $E(B-V)=0.08$.

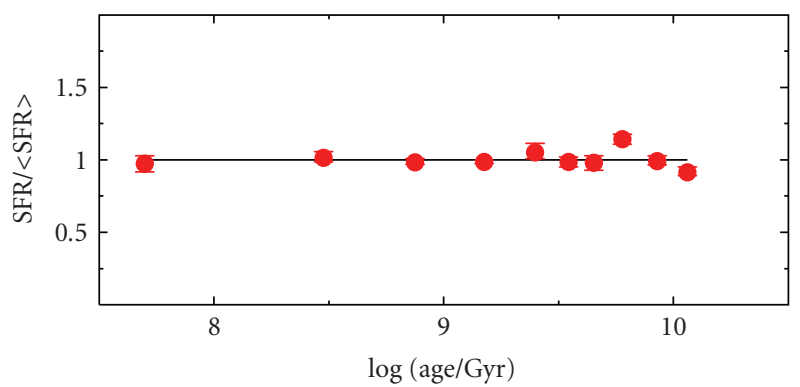

(a)

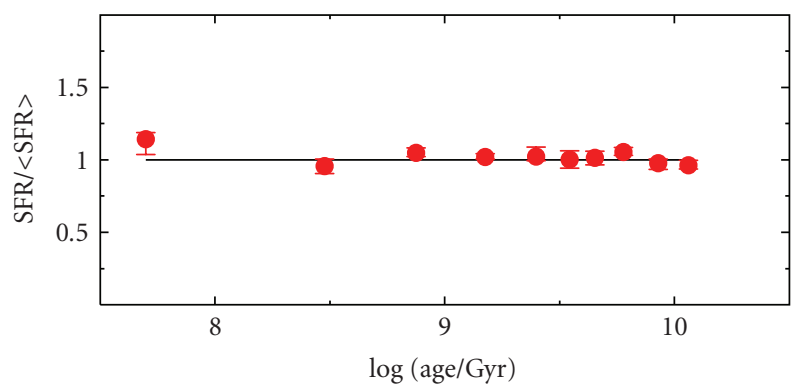

(b)

FIGURE 14: Sensitivity test to differential reddening and line-of-sight spread. Red dots represent the recovered SFH. In (a), the reference fake population is built with a distance spread $(m-M)_{0}=18.9 \pm 0.2$, while the model used to retrieve the SFH has a single distance $(m-M)_{0}=18.9$. In $(\mathrm{b})$, the reference fake population is built with a differential reddening $E(B-V)=0.08 \pm 0.04$, while the model used to retrieve the $\mathrm{SFH}$ has a single reddening $E(B-V)=0.08$.

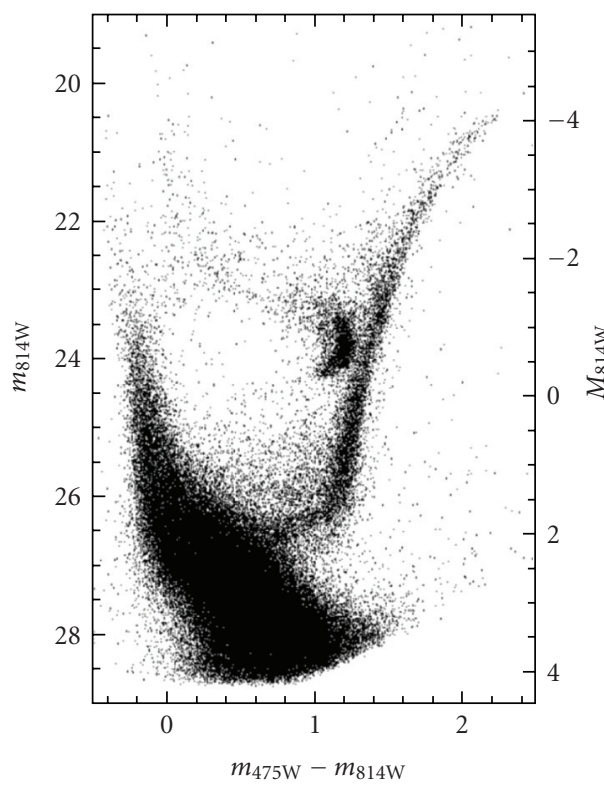

(a)

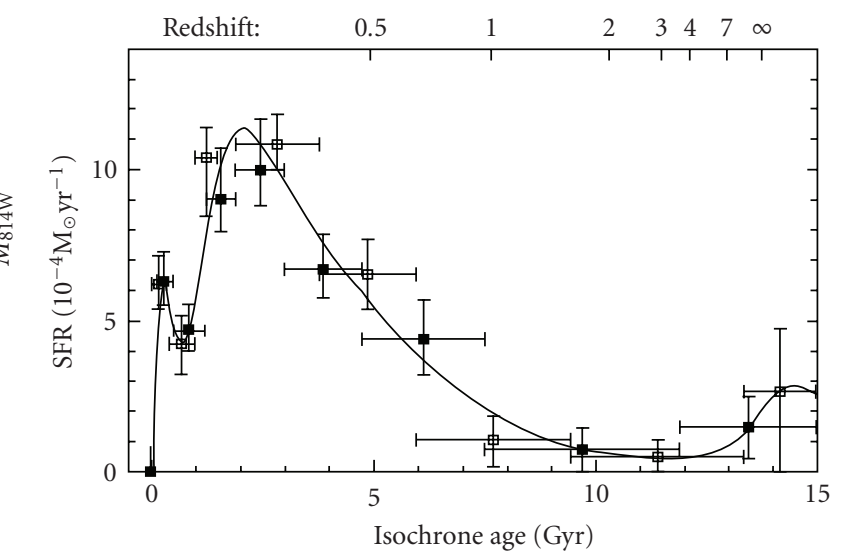

- $\quad$ Fits to CMD

- Cubic spline

(b)

FIGURE 15: CMD and SFH of Leo A as derived by [65] from HST/ACS data. Notice the impressive depth and tightness of the CMD, allowing to infer the SFH even at the earliest epochs. Courtesy A. Cole. 


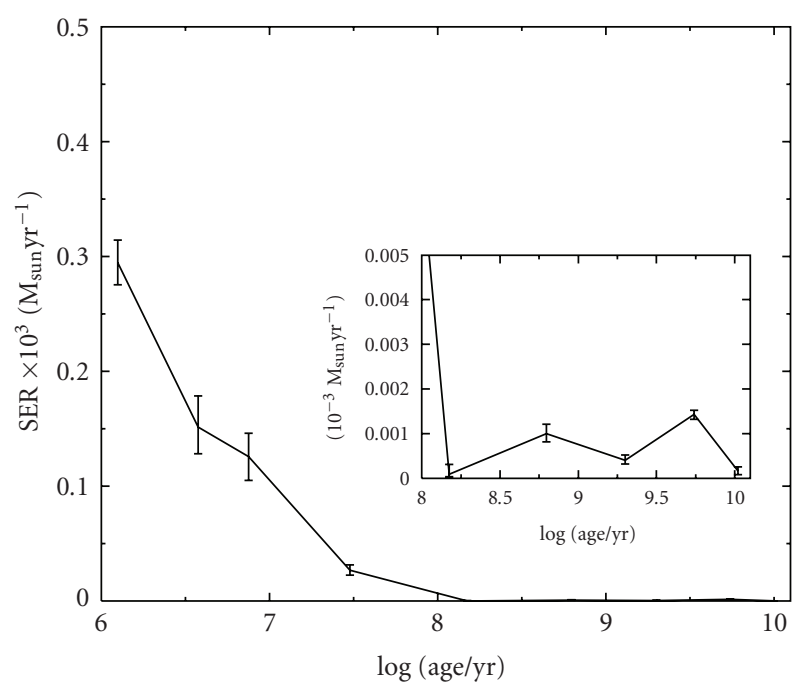

Figure 16: SFH of the ACS field centered on the SMC young cluster NGC602 as derived by [27]. The oldest part of the SFH is zoomedin in the upper right inset. The ACS image and the CMD of the field are shown in Figure 2(a).

borders of the LG. The resulting space and time distribution of the SF, with lightening and fading of adjacent cells, once again shows a gasping regime, and is intriguingly reminiscent of the predictions of the stochastic self-propagating SF theory proposed by [76] almost 30 years ago.

In the Magellanic Clouds, the conditions are clearly optimal, thanks to their proximity. Figure 16 displays the SFH of the ACS field centered on the very young cluster NGC602 in the Wing of the SMC. It shows that the cluster has formed most of its stars around $2.5 \mathrm{Myr}$ ago, while the surrounding field has formed stars continuously since the earliest epochs. All the studies on the MC fields have found that the SFHs of their different regions differ from one another in the details (e.g., epoch of activity peaks, enrichment history, etc.) but are always characterized by a gasping regime. In the LMC a clear difference has been found between the SFH of field stars and of star clusters, the latter showing a long quiescence phase absent in the field. This difference is not found in the SMC.

To find SFHs peaked at earlier epochs, one needs to look at early-type dwarfs: dwarf ellipticals (dEs), dSphs, and even transition-type dwarfs clearly underwent their major activity around or beyond $10 \mathrm{Gyr}$ ago [73]. The latter also have significat activity at recent epochs (e.g., [77]). The former have few (or no) episodes of moderate activity in the last several Gyrs (e.g., [40, 52, 78-81]). A beautiful example of CMDs and SFH of a dSph is shown in Figure 17. It is the Cetus dSph, observed with HST/ACS by the L-CID group [82] and to be published by Monelli et al. (in preparation). Here the SF activity in the last several Gyrs is negligible and the strongest peak occurred about 11 Gyr ago (interestingly, not at the earliest epoch, though).

To date, a large fraction of LG galaxies have been studied to infer the SFH of at least some of their regions with the synthetic CMD method (see, $[18,83]$ and references therein, for updated reviews): the two spirals, M31 and M33, the two Magellanic Clouds, a dozen dIrrs, 5 transition-type dwarfs and about 20 early-type dwarfs (dwarf spheroidals and dwarf ellipticals). In some of these fields, the photometry has allowed to reach the oldest MSTO, in others the HB, that is, the unmistakable signature of SF activity earlier than $10 \mathrm{Gyr}$ ago (e.g., [84-86]). Attention is being payed [87] also to the ultra-faint dwarfs (uFds) recently discovered by the Sloan Digital Sky Survey around the Milky Way (e.g., [88]), for their interest as possible Galactic building blocks. Additional efforts by various groups are in progress to obtain deeper and more accurate photometry in these and other galaxies and derive reliable SFHs over longer lookback times. For instance, very interesting results are expected from the L-CID HST program [82] on the SFH of 6 LG dwarfs of different type (two dIrrs, two dSphs, and two transition type) observed by the ACS with unprecedented depth and resolution.

In galaxies beyond the LG, distance makes crowding more severe, and even HST cannot resolve stars as faint as the MSTO of old populations. The higher the distance, the worse the crowding conditions, and the shorter the lookback time $\tau$ reachable even with the deepest, highestresolution photometry. Depending on distance and intrinsic crowding, the reachable lookback time in galaxies more than $1 \mathrm{Mpc}$ away ranges from several Gyrs (in the best cases, when the RGB or even the $\mathrm{HB}$ are clearly identified) to several hundreds Myr (when AGB stars are recognized) to a few tens Myr (when only the brightest supergiants are resolved). The effect of distance on the possibility of resolving individual stars, and therefore on the reachable $\tau$, is displayed in Figure 18, where the CMDs of three late-type galaxies are shown, as resulting from WFPC2 photometry in equivalent observing conditions: the LMC bar [68], with a distance modulus of $18.47(50 \mathrm{kpc})$ and a CMD reaching a few mags below the old MSTO; NGC1705 [91], with distance modulus $28.54(5.1 \mathrm{Mpc})$ and a CMD reaching a few mags below the tip of the RGB; and IZw18 [92]), with the new distance modulus $31.3(18 \mathrm{Mpc})$ derived by [89] and AGB stars being the faintest/older resolved stars.

Notice that the new modulus of IZw18 is inferred from the periods and luminosities of a few classical Cepheids measured from ACS time-series photometry, which also allowed to obtain a much deeper CMD [89]. The WFPC2 data shown in Figure 18 reach only the AGB, while the CMD obtained from the ACS (shown in Figure 19 together with the ACS image) reaches below the tip of the RGB. Indeed, the unique performances of the ACS have allowed people to resolve individual stars on the RGB of some of the most metal-poor Blue Compact Dwarfs (BCDs), such as SBS $1415+437$ at $13.6 \mathrm{Mpc}$ [93] and IZw18 at $18 \mathrm{Mpc}$ [89]. The discovery of stars several Gyrs old in these extremely metal-poor systems is a key information for galaxy formation and evolution studies. It has allowed to detect in BCDs population gradients, the central concentration of most of the SF activity, the existence of old, metal-poor halos (e.g., [94], and subsequent papers).

With an amazing success rate, the ACS has allowed people to resolve individual stars from the brightest and youngest to those as faint and old as the red giants in an 


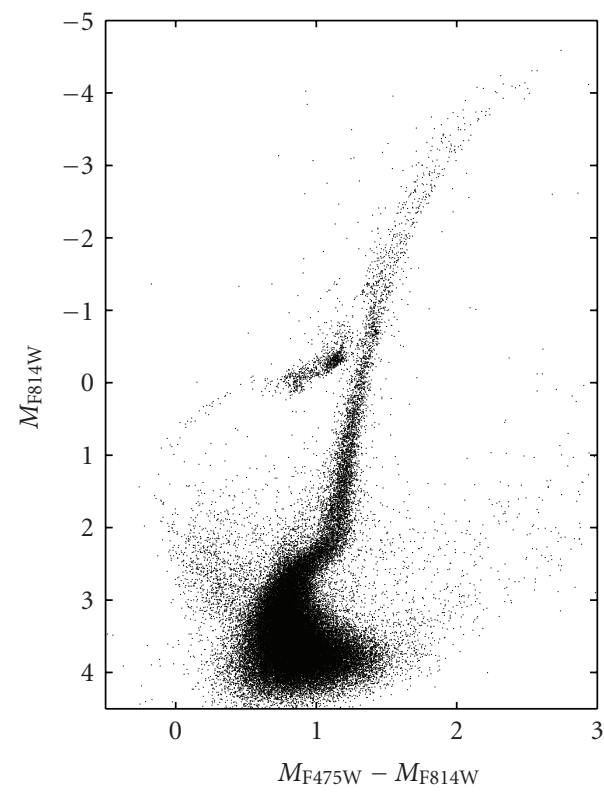

(a)

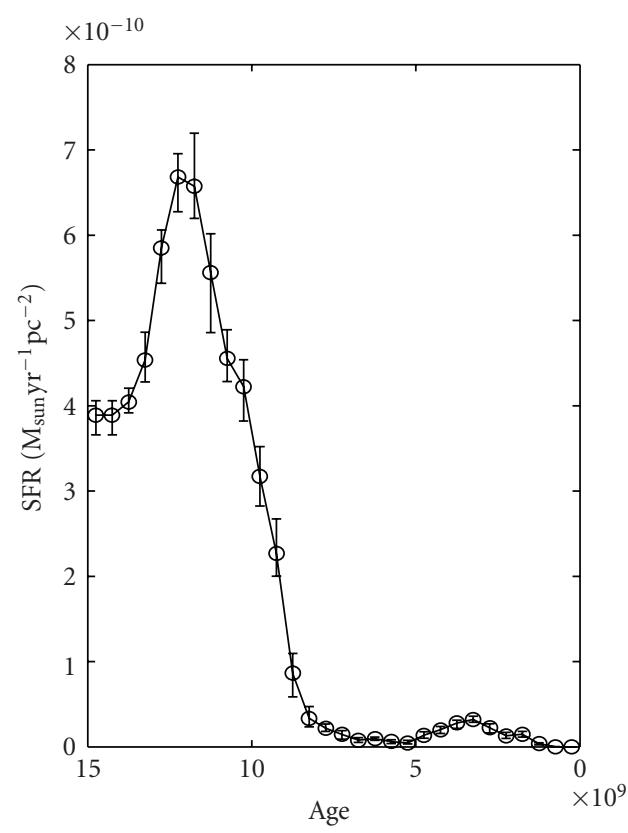

(b)

FIGURE 17: CMD and SFH of Cetus as derived by Monelli et al. (in preparation) from HST/ACS data in the framework of the L-CID project [82]. Notice again the impressive depth and tightness of the CMD, allowing to infer the SFH even at the earliest epochs. Courtesy M. Monelli and C. Gallart.

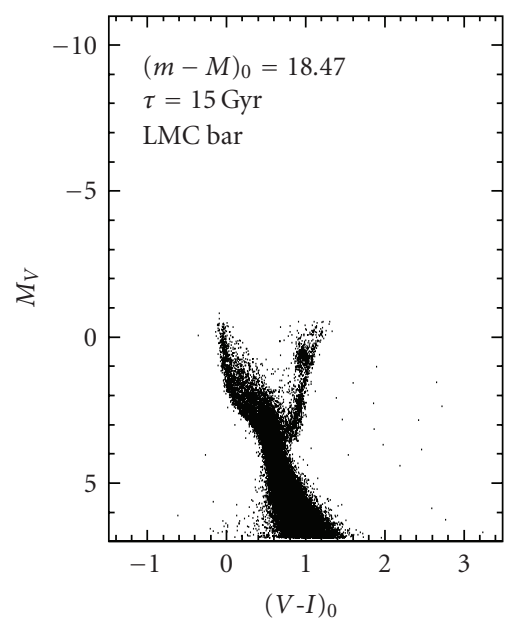

(a)

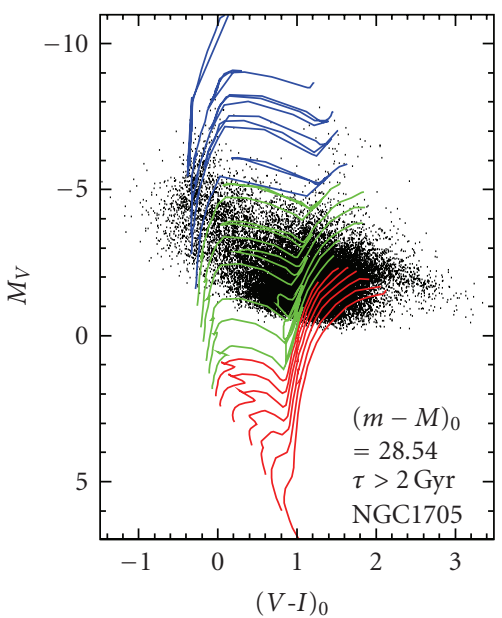

(b)

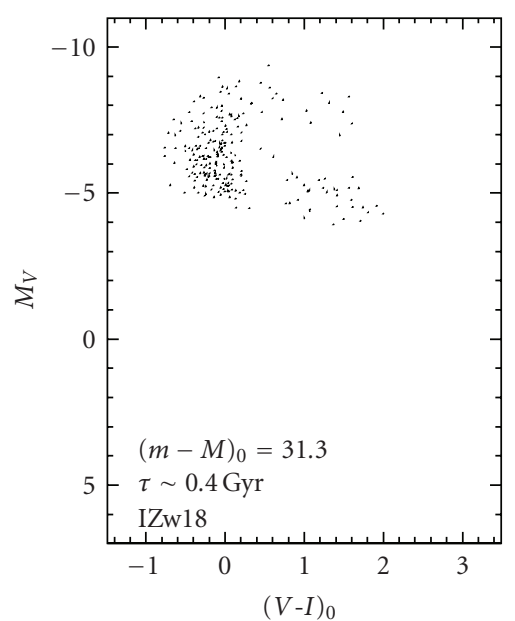

(c)

FIGURE 18: Effect of distance on the resolution of individual stars and on the corresponding lookback time $\tau$ for the SFH. CMD in absolute magnitude and colour of systems observed with the HST/WFPC2 and analysed with the same techniques, but at different distances; from (a) to (c): $50 \mathrm{Kpc}$ (LMC bar), 5.1 Mpc (NGC1705), and $18 \mathrm{Mpc}$ (IZw18); (b) also shows stellar evolution tracks from [23] for reference: red lines refer to low-mass stars, green lines to intermediate mass stars, and blue lines to massive stars.

increasing number of dwarfs outside the LG, in the distance range 2-20 Mpc. This allows to study the SFH of isolated and interacting dwarfs. People are becoming able to compare the SFHs of LG dwarfs with those of other groups of galaxies, such as the M81 (e.g., [95]) and the IC342 (Grocholski et al. in preparation) groups. The resulting CMDs lead to the derivation of their SFHs over a lookback time of at least a few
Gyrs and, often, to a more accurate estimate of their distance (e.g., $[89,96])$

Only few groups have embarked in the more challenging application of the synthetic CMD method beyond the LG, and most of them have concentrated their efforts on starbursting late-type dwarfs [92, 94, 97-99, 101-108]. However, HST (new observations and archive) is still providing a 


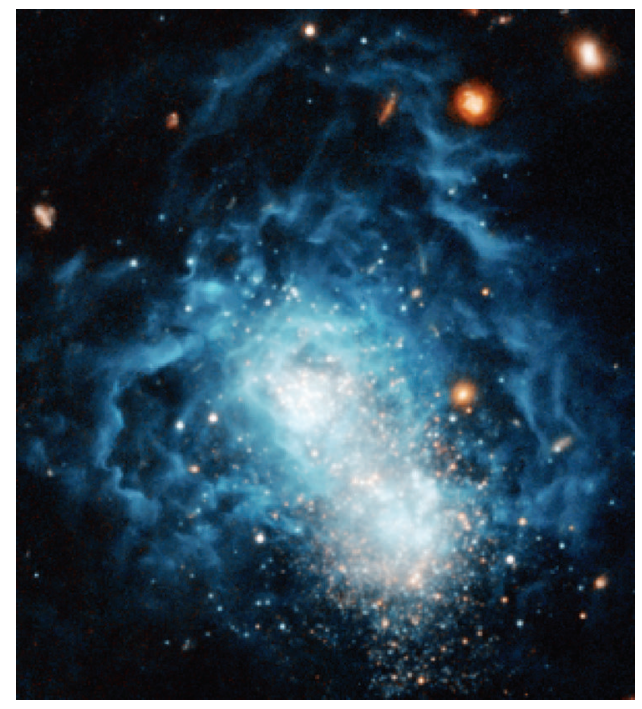

(a)

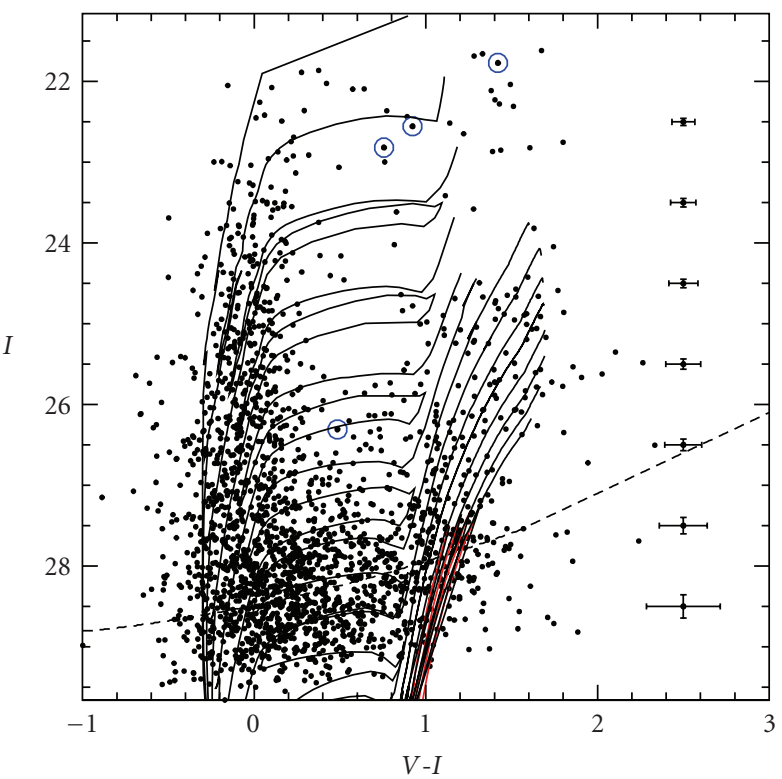

(b)

FIGURE 19: Image and CMD of IZw18, obtained from HST/ACS imaging [89]. Overimposed on the CMD are the $Z=0.0004$ isochrones by [90] with the RGB in red. Also shown is the average position of the 4 classical Cepheids with reliable light curves obtained from these data. Image credit: NASA, ESA, and A. Aloisi (STScI, ESA).

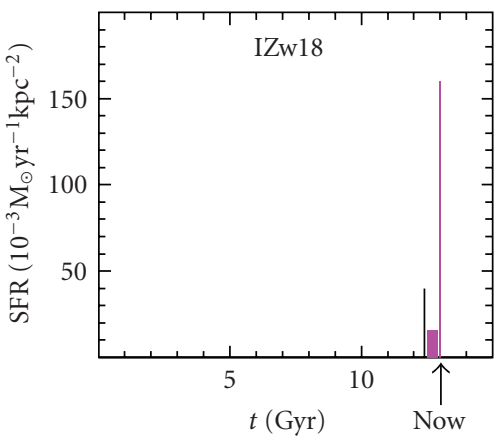

(a)

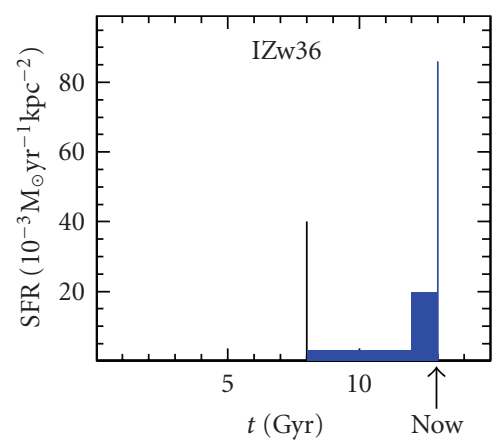

(d)

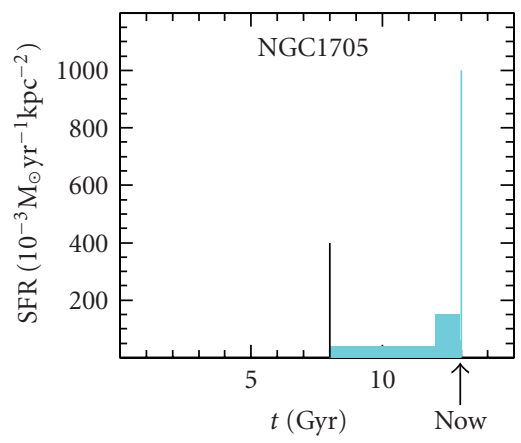

(b)

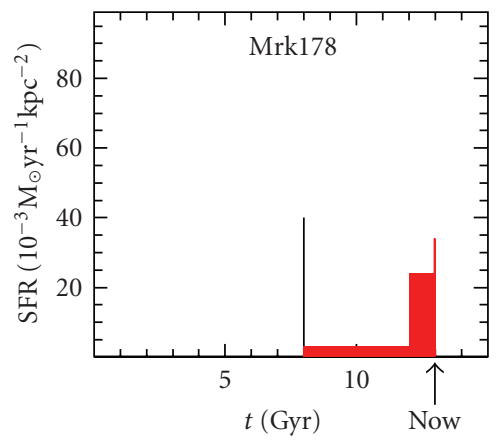

(e)

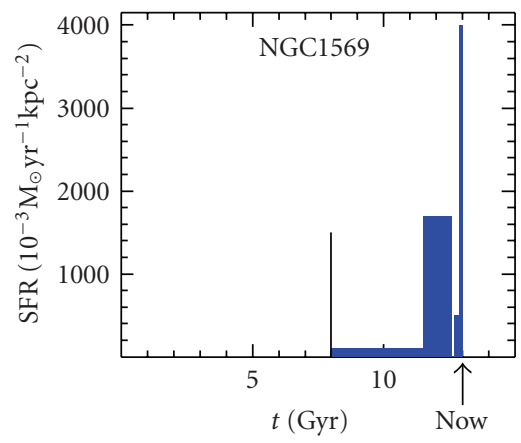

(c)

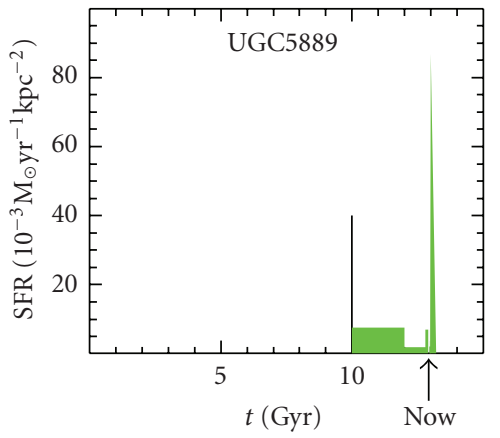

(f)

FIGURE 20: SFHs of late-type dwarfs outside the Local Group observed with WFPC2 or NICMOS. In all panels, the SFR per unit area as a function of time is plotted. The thin vertical line indicates the lookback time reached by the adopted photometry. Notice that for those galaxies that have been subsequently observed also with the ACS, the lookback time is actually quite older, and always with indisputable evidence of SF activity already in place. References: NGC 1569, [97, 98]; NGC 1705, [99, 100]; I Zw 18, [92]; I Zw 36, [101]; Mrk 178, [102]; UGC 5889, [103]. 
wealth of excellent images of external dwarfs. In particular, the ACS Nearby Galaxy Survey Treasury is promising an unprecedented data base for these purposes [109], and we can expect a flourishing of applications of the synthetic CMD method to many more galaxies beyond the LG boundaries in the near future.

All the studies performed so far have shown that all the examined galaxies were already active at the reached lookback time, including the BCDs that in the past had been suggested to be genuinely young galaxies, experiencing now their first episode of SF. All late-type dwarfs present a recent SF burst, which lets people discover them in spite of the distance, and none of them exhibits long quiescent phases within the reached lookback time. On the other hand, the SFR differs significantly from one galaxy to the other.

These results can be visualized in Figure 20, where some examples of SFH of external late-type dwarfs are given. All these SFHs have been derived with the synthetic CMD method applied to HST/WFPC2 or NICMOS photometry. The lookback time reached by the photometry is indicated by the thin vertical line in each panel, and in all cases stars of that age were detected. As shown by [100] for NGC1705, the available data allow to rule out that these galaxies have had short-duration, intermediate-age bursts like the current one within the covered lookback time. The sample of displayed galaxies contains various types of dwarfs: UGC 5889 is a low surface brightness galaxy (LSB), NGC 1705, IZw18, IZw36, and Mrk178 are BCDs, while NGC 1569 is classified as dIrr. Nonetheless, they all show a qualitatively similar behavior: a stronger current burst overimposed on a moderate and rather continuous SF activity. Quantitatively, instead, the SFRs differ from each other by orders of magnitude.

The general results drawn from all the SFHs derived so far from CMDs in and beyond the LG can be schematically summarized as follows:

(i) evidence of long interruptions in the SF activity is found only in early-type galaxies;

(ii) few early-type dwarfs have experienced only one episode of SF activity concentrated at the earliest epochs: many show instead extended or recurrent SF activity;

(iii) no galaxy currently at its first SF episode has been found yet;

(iv) no frequent evidence of strong SF bursts is found;

(v) there is no significant difference in the SFH of dIrrs and BCDs, except for the current SFR.

\section{Concluding Remarks}

By comparing the results on the SFHs described in the previous section, one can infer interesting conclusions and attempt some speculations.

An interesting result of the SFH studies both in the LG and beyond is that all dwarfs have, and have had, fairly moderate SF activity. None of the dwarfs SFRs from the CMDs studied so far ever reaches values as high as $1 M_{\odot} / \mathrm{yr}$, and only one (NGC 1569) gets close to it [96-98].
Since $1 M_{\odot} / y r$ is the minimum rate required to let a galaxy contribute to the overabundance of faint blue objects in deep galaxy counts (see the models by [2]), this makes it quite unlikely that dwarfs are responsible for the blue excess.

If we look at the dwarfs shown in Figure 20, we notice that the least active system is one of the BCDs and the most active one is the dIrr. This is not inconsistent with the findings from an extensive $H_{\alpha}$ study of 94 late-type galaxies [110], showing that the typical SFR of irregular galaxies is $10^{-3} M_{\odot} \mathrm{yr}^{-1} \mathrm{kpc}^{-2}$ and that of BCDs is generally higher. From that survey, Hunter and Elmegreen [110] indeed conclude that NGC 1569 and NGC 1705 are among the few systems with unusually high star formation, and that the star formation regions are not intrinsically different in the various galaxy types, except for a significantly higher spatial concentration in BCDs.

In our view, this suggests that either the morphological classification does not strictly correspond to the intensity of the SF activity, or that, most likely, the traditional classification, based in most cases on photographic plates, is rather uncertain for systems too distant to be properly resolved before the advent of HST. Probably an active dwarf such as NGC1569, hosting three Super Star Clusters and a huge number of HII regions, would have been classified as $\mathrm{BCD}$, had it been just a few Mpc farther away.

If we compare the SFHs of late-type dwarfs inside and outside the LG (e.g., Figures 15 and 20), we see that the overall scenario is quite similar, but the latter galaxies are always more active than the former at very recent epochs. Part of this is presumably due to the selection effect resulting from the difficulty of finding distant dwarf, faint galaxies, unless currently active. This effect is also the reason why early-type and quiescent dwarfs are so rare in the surveys performed so far outside the LG except for deep surveys devoted to individual galaxy clusters and groups, where they preferentially reside in central regions. Indeed, most often the HII region emission is what led to the discovery of distant dwarfs (recall that BCDs were originally called "extragalactic HII regions") and it is thus inevitable that these sytems have recent SF activity. From this point of view, it is interesting to note that the SFH of the external dwarfs of Figure 20 is very similar in shape to that of the NGC 602 region in the SMC [27], consistent with the circumstance that NGC 602 is also associated with an HII region (N90).

How do the Results on SFHs Affect Our Understanding of Galaxy Formation? The circumstance that all dwarfs contain stars as old as the reached lookback time, independently of their metallicity, gas content, or morphological classification suggests that their SF activity started at the earliest epochs. This is absolutely coherent with both the hierarchical formation scenario and the monolithical scenario. It is also consistent with downsizing if their early SFR was lower than that of more massive systems. For the (few) early-type dwarfs with studied SFH, we know that this is indeed the case; how about late-type dwarfs? We do not have direct evidences due to the large distance of most of these systems which prevents us to reach epochs older than a few Gyrs. However, all indirect arguments go in this direction: only 
with quite moderate early SF activity can dIrrs and BCDs have managed to remain as metal-poor and gas-rich as they actually are. SFRs as high as the recent ones would have inevitably consumed all their gas in much less than a Hubble time and would have led to a significant chemical enrichment.

As mentioned in Section 1, to select the most viable scenario, it is the combination of the SFH with the chemical and kinematic properties of the candidate building blocks that needs to be compared with the properties of massive galaxies. In the case of local dwarfs, these properties have been recently reviewed by [18]. Stars in classical dwarfs do not resemble those in the halo of the Milky Way, most notably their metallicity distribution functions [111-113] and the abundance ratios of $\alpha$ elements over iron (see [18], and references therein). Moreover, if all early-type dwarfs have had the relatively moderate SF activity shown in Figure 17 for Cetus, with a rather long duration and the peak some Gyr after the beginning, there is no way to let them provide the iron-poor stars with high $[\alpha / \mathrm{Fe}]$ typical of our halo, since the SF peak forms most of the stars when SNeIa have already had the time to pollute the medium with their iron.

On the other hand, the current knowledge of the outer Galaxy is far from complete. We know that the stellar halo hosts two distinct populations (see, e.g., $[114,115]$, and references therein). The work in [116] finds that the socalled "inner halo" selected among halo stars with prograde rotation and low apogalactic maximum distance from the galactic center is different for several aspects from the "outer halo" selected among stars with high retrograde rotation and high apogalactic maximum distance. In particular, the following hold. (1) The inner halo is characterized by a tight correlation between $[\alpha / F e]$ versus $[F e / H]$, suggesting that either the abundance ratios in distant regions of the inner halo are very similar or the inner halo developed from a well-homogenized interstellar medium. In contrast, the outer halo shows a much larger scatter in $[\alpha / F e]$ for a given $[\mathrm{Fe} / \mathrm{H}]$, signature that the star formation was spatially inhomogeneous or these stars have been accreted from outside (from dwarf galaxies). (2) The inner halo shows an average $[\alpha / F e]$ slightly higher than observed in the outer halo, providing a clue for a more intense and short-lasting star formation activity. (3)The inner halo is only found with metallicities in the range $-2.5<[\mathrm{Fe} / \mathrm{H}]<-0.5$, while most of the outer halo is in the range $-3.5<[\mathrm{Fe} / \mathrm{H}]<-1.5$.

Unfortunately, the outer halo is still mostly inaccessible: current high-resolution abundances rely mainly on halo stars that pass near the Sun. If these stars are formed in the outer halo, their selection is biased towards higher eccentricity orbits. Avoiding this bias implies a new class of surveys able to trace $\alpha$ variations in situ. In this context, the Gaia mission will provide a quantum leap in the ability to obtain highly precise astrometry, photometry and metallicity for a volume of several Kpc.

If the outer halo is a natural place to search for possible accretion events, the recent discovery of ultrafaint dwarfs promises to complete the picture: containing extremely metal-poor stars, probably with high $[\alpha / \mathrm{Fe}]$ like in our halo [117-119], these galaxies are ideal candidates for Galactic building blocks. The problem in this case is the extreme uncertainty still affecting their measures, due to both faintness and high Galactic contamination. Overcoming these limits will be a challenge only suitable for wide-field spectrographs mounted on giant ground-based telescopes.

\section{Acknowledgments}

Over the years several interesting conversations with A. Aparicio, C. Chiosi, S. DeglâInnocenti, J. Gallagher, C. Gallart, P.G. Prada Moroni, R. Schulte-Ladbeck, S. N. Shore, E. Skillman, E. Tolstoy, and in particular L. Greggio, have been fruitful to dig into the secrets of synthetic CMD building and exploitation. We thank A. Cole for providing the Leo A figure in appropriate format and M. Monelli for the Cetus figure. We also thank Laura Greggio and the anonymous referees for detailed and constructive comments helpful to make this paper clearer. We acknowledge the financial support from ASI through contract ASI-INAF I/016/07/0 and from the Italian MIUR through contract PRIN-2007JJC53X-001.

\section{References}

[1] S. J. Lilly, L. Tresse, F. Hammer, D. Crampton, and O. Le Fèvre, "The canada-france redshift survey. VI. Evolution of the galaxy luminosity function to $\mathrm{z}$ approximately 1," The Astrophysical Journal, vol. 455, no. 1, pp. 108-124, 1995.

[2] A. Babul and H. C. Ferguson, "Faint blue galaxies and the epoch of dwarf galaxy formation," The Astrophysical Journal, vol. 458, no. 1, pp. 100-119, 1996.

[3] M. Peimbert and S. Torres-Peimbert, "Chemical composition of H II regions in the Large Magellanic Cloud and its cosmological implications," The Astrophysical Journal, vol. 193, pp. 327-333, 1974.

[4] K. A. Olive, G. Steigman, and E. D. Skillman, "The primordial abundance of4He: an update," The Astrophysical Journal, vol. 483, no. 2, part 1, pp. 788-797, 1997.

[5] Y. I. Izotov and T. X. Thuan, "The primordial abundance of 4He revisited," The Astrophysical Journal, vol. 500, p. 188, 1998.

[6] S. D. M. White and M. J. Rees, "Core condensation in heavy halos-a two-stage theory for galaxy formation and clustering," Monthly Notices of the Royal Astronomical Society, vol. 183, pp. 341-358, 1978.

[7] C. S. Frenk, S. D. M. White, M. Davis, and G. Efstathiou, "The formation of dark halos in a universe dominated by cold dark matter," The Astrophysical Journal, vol. 327, pp. 507-525, 1988.

[8] M. Bellazzini, R. Ibata, F. R. Ferraro, and V. Testa, "Tracing the Sgr stream with 2MASS: detection of stream stars around outer halo globular clusters," Astronomy and Astrophysics, vol. 405, no. 2, pp. 577-583, 2003.

[9] V. Belokurov, D. B. Zucker, N. W. Evans, et al., "Cats and dogs, hair and a hero: a quintet of new milky way companions," Astrophysical Journal, vol. 654, no. 2, pp. 897906, 2007.

[10] A. M. N. Ferguson, M. J. Irwin, R. A. Ibata, G. F. Lewis, and N. R. Tanvir, "Evidence for stellar substructure in the halo 
and outer disk of M31," The Astronomical Journal, vol. 124, pp. 1452-1463, 2002.

[11] R. Ibata, S. Chapman, A. M. N. Ferguson, M. Irwin, G. Lewis, and A. McConnachie, "Taking measure of the Andromeda halo: a kinematic analysis of the giant stream surrounding M31," Monthly Notices of the Royal Astronomical Society, vol. 351, no. 1, pp. 117-124, 2004.

[12] A. M. N. Ferguson, R. A. Johnson, D. C. Faria, et al., "The stellar populations of the M31 Halo substructure," The Astrophysical Journal, vol. 622, no. 2, part 2, pp. L109-L112, 2005.

[13] L. L. Cowie, A. Songaila, E. M. Hu, and J. G. Cohen, "New insight on galaxy formation and evolution from keck spectroscopy of the Hawaii deep fields," The Astronomical Journal, vol. 112, no. 3, pp. 839-864, 1996.

[14] H. Mouri and Y. Taniguchi, "Downsizing of star-forming galaxies by gravitational processes," Astronomy and Astrophysics, vol. 459, no. 2, pp. 371-374, 2006.

[15] E. Neistein, F. C. van den Bosch, and A. Dekel, "Natural downsizing in hierarchical galaxy formation," Monthly Notices of the Royal Astronomical Society, vol. 372, no. 2, pp. 933-948, 2006.

[16] A. Cattaneo, A. Dekel, S. M. Faber, and B. Guiderdoni, "Downsizing by shutdown in red galaxies," Monthly Notices of the Royal Astronomical Society, vol. 389, no. 2, pp. 567-584, 2008.

[17] O. J. Eggen, D. Lynden-Bell, and A. R. Sandage, "Evidence from the motions of old stars that the galaxy collapsed," The Astrophysical Journal, vol. 136, p. 748, 1962.

[18] E. Tolstoy, V. Hill, and M. Tosi, "Star formation histories, abundances and kinematics of dwarf galaxies in the local group," Annual Review of Astronomy and Astrophysics, vol. 47, pp. 371-425, 2009.

[19] L. Searle, W. L. W. Sargent, and W. G. Bagnuolo, "The history of star formation and the colors of late-type galaxies," The Astrophysical Journal, vol. 179, pp. 427-438, 1973.

[20] J. S. Gallagherc III, D. A. Hunter, and A. V. Tutukov, "Star formation histories of irregular galaxies," The Astrophysical Journal, vol. 284, pp. 544-556, 1984.

[21] J. S. Gallagher III and D. A. Hunter, "Structure and evolution of irregular galaxies," Annual Review of Astronomy and Astrophysics, vol. 22, pp. 37-74, 1984.

[22] F. Fagotto, A. Bressan, G. Bertelli, and C. Chios, "Evolutionary sequences of stellar models with new radiative opacities. $\mathrm{Z}=.0004$ and $\mathrm{Z}=.05$," Astronomy and Astrophysics Supplement Series, vol. 104, pp. 365-376, 1996.

[23] F. Fagotto, A. Bressan, G. Bertelli, and C. Chios, "Evolutionary sequences of stellar models with new radiative opacities. VI. $\mathrm{Z}=.004$ and $\mathrm{Z}=.008$," Astronomy and Astrophysics Supplement Series, vol. 105, pp. 29-38, 1996.

[24] L. Greggio, "The color-magnitude diagram of composite stellar populations," in Observed HR Diagrams and Stellar Evolution, T. Lejeune and J. Fernandes, Eds., vol. 274 of Astronomical Society of the Pacific Conference Series, p. 444, 2002.

[25] L. R. Carlson, E. Sabbi, M. Sirianni, et al., "Progressive star formation in the young SMC cluster NGC 602," The Astrophysical Journal, vol. 665, no. 2, part 2, pp. L109-L114, 2007.

[26] E. Sabbi, M. Sirianni, A. Nota, et al., "Past and present star formation in the SMC: NGC 346 and its neighborhood," The Astronomical Journal, vol. 133, no. 1, pp. 44-57, 2007.
[27] M. Cignoni, E. Sabbi, A. Nota, et al., "Star formation history in the small magellanic cloud: the case of NGC 602," The Astronomical Journal, vol. 137, no. 3, pp. 3668-3684, 2009.

[28] P. Kroupa, "On the variation of the initial mass function," Monthly Notices of the Royal Astronomical Society, vol. 322, no. 2, pp. 231-246, 2001.

[29] G. Chabrier, "Galactic stellar and substellar initial mass function," Publications of the Astronomical Society of the Pacific, vol. 115, no. 809, pp. 763-795, 2003.

[30] F. R. Ferraro, F. Fusi Pecci, M. Tosi, and R. Buonanno, "A method for studying the star formation history of dwarf irregular galaxies. I - CCD photometry of WLM," Monthly Notices of the Royal Astronomical Society, vol. 241, pp. 433452, 1989.

[31] M. Tosi, L. Greggio, G. Marconi, and P. Focardi, "Star formation in dwarf irregular galaxies: sextans B," The Astronomical Journal, vol. 102, no. 3, pp. 951-974, 1991.

[32] G. Bertelli, M. Mateo, C. Chiosi, and A. Bressan, "The star formation history of the large magellanic cloud," The Astrophysical Journal, vol. 388, no. 2, pp. 400-414, 1992.

[33] L. Greggio, G. Marconi, M. Tosi, and P. Focardi, "Star formation in dwarf irregular galaxies-DDO 210 and NGC 3109," The Astronomical Journal, vol. 105, no. 3, pp. 894-932, 1993.

[34] G. Marconi, M. Tosi, L. Greggio, and P. Focardi, "Star formation in dwarf irregular galaxies: NGC 6822," The Astronomical Journal, vol. 109, no. 1, pp. 173-199, 1995.

[35] C. Gallart, A. Aparicio, G. Bertelli, and C. Chiosi, "The Local Group dwarf irregular galaxy NGC 6822. II. The old and intermediate-age star formation history," The Astronomical Journal, vol. 112, no. 5, pp. 1950-1968, 1996.

[36] C. Gallart, A. Aparicio, G. Bertelli, and C. Chiosi, “The local group dwarf irregular galaxy NGC 6822. III. The recent star formation history," The Astronomical Journal, vol. 112, no. 6, pp. 2596-2606, 1996.

[37] A. Aparicio, C. Gallart, and G. Bertelli, "The star formation history of the pegasus dwarf irregular galaxy," The Astronomical Journal, vol. 114, no. 2, pp. 669-679, 1997.

[38] E. Tolstoy and A. Saha, "The interpretation of colormagnitude diagrams through numerical simulation and bayesian inference," The Astrophysical Journal, vol. 462, no. 2, part 1, pp. 672-683, 1996.

[39] A. Dolphin, "A new method to determine star formation histories of nearby galaxies," New Astronomy, vol. 2, no. 5, pp. 397-409, 1997.

[40] C. Gallart, W. L. Freedman, A. Aparicio, G. Bertelli, and C. Chiosi, "The star formation history of the local group dwarf galaxy Leo I," The Astronomical Journal, vol. 118, no. 5, pp. 2245-2261, 1999.

[41] A. A. Cole, E. Tolstoy, J. S. Gallagher III, et al., "Stellar populations at the center of IC 1613," The Astronomical Journal, vol. 118, no. 4, pp. 1657-1670, 1999.

[42] X. Hernandez, D. Valls-Gabaud, and G. Gilmore, "Deriving star formation histories: inverting Hertzsprung-Russell diagrams through a variational calculus maximum likelihood method," Monthly Notices of the Royal Astronomical Society, vol. 304, no. 4, pp. 705-719, 1999.

[43] J. A. Holtzman, J. S. Gallagher III, A. A. Cole, et al., "Observations and implications of the star formation history of the large magellanic cloud," The Astronomical Journal, vol. 118, no. 5, pp. 2262-2279, 1999. 
[44] J. Harris and D. Zaritsky, "A method for determining the star formation history of a mixed stellar population," Astrophysical Journal, Supplement Series, vol. 136, no. 1, pp. 25-40, 2001.

[45] J.-L. Vergely, J. Köppen, D. Egret, and O. Bienaymé, "An inverse method to interpret colour-magnitude diagrams," Astronomy and Astrophysics, vol. 390, no. 3, pp. 917-929, 2002.

[46] M. Cignoni, S. Degl'Innocenti, P. G. Prada Moroni, and S. N. Shore, "Recovering the star formation rate in the solar neighborhood," Astronomy and Astrophysics, vol. 459, no. 3, pp. 783-796, 2006.

[47] K.-P. Schröder and B. E. J. Pagel, "Galactic archaeology: initial mass function and depletion in the 'thin disc'," Monthly Notices of the Royal Astronomical Society, vol. 343, no. 4, pp. 1231-1240, 2003.

[48] X. Hernandez, D. Valls-Gabaud, and G. Gilmore, "The recent star formation history of the Hipparcos solar neighbourhood," Monthly Notices of the Royal Astronomical Society, vol. 316, no. 3, pp. 605-612, 2000.

[49] T. Naylor and R. D. Jeffries, "A maximum-likelihood method for fitting colour-magnitude diagrams," Monthly Notices of the Royal Astronomical Society, vol. 373, no. 3, pp. 1251-1263, 2006.

[50] E. D. Skillman and C. Gallart, "First results of the coimbra experiment," in Observed HR Diagrams and Stellar Evolution, T. Lejeune and J. Fernandes, Eds., vol. 274 of Astronomical Society of the Pacific Conference Series, p. 535, 2002.

[51] M. Cignoni, S. Degl'Innocenti, P. G. Prada Moroni, and S. N. Shore, "Recovering the star formation rate in the solar neighborhood," Astronomy and Astrophysics, vol. 459, no. 3, pp. 783-796, 2006.

[52] A. E. Dolphin, "Numerical methods of star formation history measurement and applications to seven dwarf spheroidals," Monthly Notices of the Royal Astronomical Society, vol. 332, no. 1, pp. 91-108, 2002.

[53] A. Aparicio and S. L. Hidalgo, "IAC-pop: finding the star formation history of resolved galaxies," The Astronomical Journal, vol. 138, no. 2, pp. 558-567, 2009.

[54] C. Gallart, M. Zoccali, and A. Aparicio, "The adequacy of stellar evolution models for the interpretation of the colormagnitude diagrams of resolved stellar populations," Annual Review of Astronomy and Astrophysics, vol. 43, pp. 387-434, 2005.

[55] L. O. Kerber, L. Girardi, S. Rubele, and M.-R. Cioni, "Recovery of the star formation history of the LMC from the VISTA survey of the Magellanic system," Astronomy and Astrophysics, vol. 499, no. 3, pp. 697-710, 2009.

[56] A. Aparicio, C. Gallart, and G. Bertelli, "The stellar content and the star formation history of the local group dwarf galaxy LGS 3," The Astronomical Journal, vol. 114, no. 2, pp. 680693, 1997.

[57] J. Harris and D. Zaritsky, "The star formation history of the small magellanic cloud," The Astronomical Journal, vol. 127, no. 3 1779, pp. 1531-1544, 2004.

[58] E. E. Salpeter, "The luminosity function and stellar evolution,” The Astrophysical Journal, vol. 121, p. 161, 1955.

[59] B. M. Tinsley, "Evolution of the stars and gas in galaxies," Fundamentals of Cosmic Physics, vol. 5, pp. 287-388, 1980.

[60] J. Scalo, "The IMF revisited: a case for variations," in The Stellar Initial Mass Function (38th Herstmonceux Conference),
G. Gilmore and D. Howell, Eds., vol. 142 of Astronomical Society of the Pacific Conference Series, p. 201, 1998.

[61] J. Hurley and C. A. Tout, "The binary second sequence in cluster colour-magnitude diagrams," Monthly Notices of the Royal Astronomical Society, vol. 300, no. 4, pp. 977-980, 1998.

[62] K. A. G. Olsen, "Star formation histories from hubble space telescope color-magnitude diagrams of six fields of the large magellanic cloud," The Astronomical Journal, vol. 117, no. 5, pp. 2244-2267, 1999.

[63] S. Stanimirović, L. Staveley-Smith, and P. A. Jones, "A new look at the kinematics of neutral hydrogen in the small magellanic cloud," Astrophysical Journal, vol. 604, pp. 176186, 2004.

[64] E. D. Skillman, E. Tolstoy, A. A. Cole, et al., "Deep hubble space telescope imaging of IC 1613. II. The star formation history," Astrophysical Journal, vol. 596, pp. 253-272, 2003.

[65] A. A. Cole, E. D. Skillman, E. Tolstoy, et al., "Leo A: a lateblooming survivor of the epoch of reionization in the local group," The Astrophysical Journal, vol. 659, no. 1, part 2, pp. L17-L20, 2007.

[66] E. Tolstoy, "The resolved stellar population of leo A," The Astrophysical Journal, vol. 462, no. 2, part 1, pp. 684-704, 1996.

[67] A. E. Dolphin, "The star formation histories of two northern LMC fields," Monthly Notices of the Royal Astronomical Society, vol. 313, no. 2, pp. 281-290, 2000.

[68] T. A. Smecker-Hane, A. A. Cole, J. S. Gallagher III, and P. B. Stetson, "The star formation history of the large magellanic cloud," The Astrophysical Journal, vol. 566, no. 1, part 1, pp. 239-244, 2002.

[69] S. C. Javiel, B. X. Santiago, and L. O. Kerber, "Constraints on the star formation history of the large magellanic cloud," Astronomy and Astrophysics, vol. 431, no. 1, pp. 73-85, 2005.

[70] C. Gallart, P. B. Stetson, I. P. Meschin, F. Pont, and E. Hardy, "Outside-in disk evolution in the large magellanic cloud," The Astrophysical Journal, vol. 682, no. 2, part 2, pp. L89-L92, 2008.

[71] A. E. Dolphin, A. R. Walker, P. W. Hodge, et al., "Old stellar populations of the small magellanic cloud," The Astrophysical Journal, vol. 562, no. 1 , part 1, pp. 303-313, 2001.

[72] N. E. D. Noël, C. Gallart, E. Costa, and R. A. Mèndez, "Old main-sequence turnoff photometry in the small magellanic cloud. I. Constraints on the star formation history in different fields," The Astronomical Journal, vol. 133, no. 5, pp. 2037-2052, 2007.

[73] S. L. Hidalgo, A. Aparicio, and C. Gallart, "Recovering the ages and metallicities of stars of a complex stellar population system," in The Ages of Stars, E. E. Mamajek, D. R. Soderblom, and R. F. G. Wyse, Eds., vol. 258 of International Astronomical Union Symposium, pp. 245-252, June 2009.

[74] R. C. Dohm-Palmer, E. D. Skillman, J. Gallagher, et al., "The recent star formation history of GR 8 from hubble space telescope photometry of the resolved stars," The Astronomical Journal, vol. 116, no. 3, pp. 1227-1243, 1998.

[75] R. C. Dohm-Palmer, E. D. Skillman, M. Mateo, et al., "Deep hubble space telescope imaging of Sextans A. I. The spatially resolved recent star formation history," The Astronomical Journal, vol. 123, no. 2, pp. 813-831, 2002.

[76] P. E. Seiden, L. S. Schulman, and H. Gerola, "Stochastic star formation and the evolution of galaxies," The Astrophysical Journal, vol. 232, pp. 702-706, 1979. 
[77] L. M. Young, E. D. Skillman, D. R. Weisz, and A. E. Dolphin, "The aptly named phoenix dwarf galaxy," The Astrophysical Journal, vol. 659, no. 1, part 1, pp. 331-338, 2007.

[78] T. A. Smecker-Hane, P. B. Stetson, J. E. Hesser, and D. A. Vandenberg, "Episodic star formation in the carina dSph galaxy," in From Stars to Galaxies: The Impact of Stellar Physics on Galaxy Evolution, C. Leitherer, U. Fritze-von-Alvensleben, and J. Huchra, Eds., vol. 98 of Astronomical Society of the Pacific Conference Series, pp. 328-332, 1996.

[79] D. Hurley-Keller, M. Mateo, and J. Nemec, "The star formation history of the Carina dwarf galaxy," The Astronomical Journal, vol. 115, no. 5, pp. 1840-1855, 1998.

[80] X. Hernandez, G. Gilmore, and D. Valls-Gabaud, "Nonparametric star formation histories for four dwarf spheroidal galaxies of the local group," Monthly Notices of the Royal Astronomical Society, vol. 317, no. 4, pp. 831-842, 2000.

[81] A. E. Dolphin, D. R. Weisz, E. D. Skillman, and J. A. Holtzman, "Star formation histories of local group dwarf galaxies," Astrophysics and Space Science, vol. 284, no. 2, pp. 589-594, 2003.

[82] C. Gallart and The Lcid Team, "The ACS LCID project: overview and first results," in Proceedings of the International Astronomical Union, A. Vazdekis and R. F. Peletier, Eds., vol. 241 of International Astronomical Union Symposium, pp. 290-294, August 2007.

[83] M. Tosi, "Star formation histories of resolved galaxies," in Proceedings of the International Astronomical Union, E. E. Mamajek, D. R. Soderblom, and R. F. G. Wyse, Eds., vol. 258 of International Astronomical Union Symposium, pp. 61-72, June 2009.

[84] E. V. Held, I. Saviane, Y. Momany, and G. Carraro, "The elusive old population of the dwarf spheroidal galaxy Leo I," The Astrophysical Journal, vol. 530, no. 2, part 2, pp. L85-L88, 2000.

[85] L. Baldacci, L. Rizzi, G. Clementini, and E. V. Held, "Variable stars in the dwarf irregular galaxy NGC 6822: thephotometric catalogue," Astronomy \& Astrophysics, vol. 431, pp. 11891201, 2005.

[86] Y. Momany, E. V. Held, I. Saviane, et al., "HST/ACS observations of the old and metal-poor Sagittarius dwarf irregular galaxy," Astronomy and Astrophysics, vol. 439, no. 1, pp. 111-127, 2005.

[87] J. T. A. de Jong, J. Harris, M. G. Coleman, et al., "The structural properties and star formation history of Leo $\mathrm{T}$ from deep LBT photometry," The Astrophysical Journal, vol. 680, no. 2, pp. 1112-1119, 2008.

[88] V. Belokurov, M. G. Walker, N. W. Evans, et al., "Leo V: a companion of a companion of the milky way galaxy?" The Astrophysical Journal, vol. 686, pp. L83-L86, 2008.

[89] A. Aloisi, G. Clementini, M. Tosi, et al., "I Zw 18 revisited with HST ACS and Cepheids: new distance and age," The Astrophysical Journal, vol. 667, no. 2, part 2, pp. L151-L154, 2007.

[90] G. Bertelli, A. Bressan, C. Chiosi, F. Fagotto, and E. Nasi, "Theoretical isochrones from models with new radiative opacities," Astronomy \& Astrophysics, vol. 106, pp. 275-302, 1994.

[91] M. Tosi, E. Sabbi, M. Bellazzini, et al., "The resolved stellar populations in NGC 1705," The Astronomical Journal, vol. 122, no. 3, pp. 1271-1288, 2001.

[92] A. Aloisi, M. Tosi, and L. Greggio, "The star formation history of I Zw 18," The Astronomical Journal, vol. 118, no. 1, pp. 302-322, 1999.
[93] A. Aloisi, R. P. van der Marel, J. Mack, C. Leitherer, M. Sirianni, and M. Tosi, "Do young galaxies exist in the local universe? red giant branch detection in the metal-poor dwarf galaxy SBS 1415+437," The Astrophysical Journal, vol. 631, pp. L45-L48, 2005.

[94] R. E. Schulte-Ladbeck, U. Hopp, L. Greggio, and M. M. Crone, "A near-infrared stellar census of the blue compact dwarf galaxy VII Zw 403," The Astronomical Journal, vol. 118, no. 6, pp. 2705-2722, 1999.

[95] D. R. Weisz, E. D. Skillman, J. M. Cannon, et al., "The recent star formation histories of M81 group dwarf irregular galaxies," The Astrophysical Journal, vol. 689, no. 1, pp. 160183, 2008.

[96] A. J. Grocholski, A. Aloisi, R. P. van der Marel, et al., "A new hubble space telescope distance to NGC 1569: starburst properties and IC 342 group membership," The Astrophysical Journal, vol. 686, no. 2, part 2, pp. L79-L82, 2008.

[97] L. Gregoio, M. Tosi, et al., "The resolved stellar population of the poststarburst galaxy NGC 1569," The Astrophysical Journal, vol. 504, no. 2, part 1, pp. 725-742, 1998.

[98] L. Angeretti, M. Tosi, L. Greggio, E. Sabbi, A. Aloisi, and C. Leitherer, "The complex star formation history of NGC 1569," The Astronomical Journal, vol. 129, no. 5, pp. 22032216, 2005.

[99] F. Annibali, L. Greggio, M. Tosi, A. Aloisi, and C. Leitherer, "The star formation history of NGC 1705: a poststarburst galaxy on the verge of activity," The Astronomical Journal, vol. 126, pp. 2752-2773, 2003.

[100] F. Annibali, M. Tosi, M. Monelli, et al., "Young stellar populations and star clusters in NGC 1705," The Astronomical Journal, vol. 138, no. 1, pp. 169-183, 2009.

[101] R. E. Schulte-Ladbeck, U. Hopp, L. Greggio, M. M. Crone, and I. O. Drozdovsky, "A near-infrared stellar census of blue compact dwarf galaxies: the Wolf-Rayet galaxy I Zw 36," The Astronomical Journal, vol. 121, no. 6, pp. 3007-3025, 2001.

[102] R. E. Schulte-Ladbeck, U. Hopp, L. Greggio, and M. M. Crone, "A near-infrared stellar census of blue compact dwarf galaxies: nicmos detection of red giant stars in the Wolf-Rayet galaxy Markarian 178," Astronomical Journal, vol. 120, no. 4, pp. 1713-1730, 2000.

[103] A. Vallenari, L. Schmidtobreick, and D. J. Bomans, “The star formation history of the LSB galaxy UGC 5889," Astronomy and Astrophysics, vol. 435, no. 3, pp. 821-829, 2005.

[104] A. Vallenari and D. J. Bomans, "Star formation history of the post starburst galaxy NGC 1569," Astronomy and Astrophysics, vol. 313, no. 3, pp. 713-722, 1996.

[105] R. Lynds, E. Tolstoy, E. J. O’Neil Jr., and D. A. Hunter, “Star formation in and evolution of the blue compact dwarf galaxy UGC 6456 determined from Hubble space telescope images," The Astronomical Journal, vol. 116, no. 1, pp. 146-162, 1998.

[106] A. E. Dolphin, L. Makarova, I. D. Karachentsev, et al., "The stellar content and distance of UGC 4483," Monthly Notices of the Royal Astronomical Society, vol. 324, no. 1, pp. 249-256, 2001.

[107] M. M. Crone, R. E. Schulte-Ladbeck, L. Greggio, and U. Hopp, "The star formation history of the blue compact dwarf galaxy UGCA 290," The Astrophysical Journal, vol. 567, no. 1 ,part 1, pp. 258-276, 2002.

[108] K. B. W. McQuinn, E. D. Skillman, J. M. Cannon, et al., "The true durations of starbursts: HST observations of three nearby dwarf starburst galaxies," The Astrophysical Journal, vol. 695 , no. 1, pp. 561-573, 2009. 
[109] B. F. Williams, J. J. Dalcanton, A. C. Seth, et al., "The acs nearby galaxy survey treasury. I. the star formation history of the M81 outer disk," The Astronomical Journal, vol. 137, no. 1, pp. 419-430, 2009.

[110] D. A. Hunter and B. G. Elmegreen, "Star formation properties of a large sample of irregular galaxies," The Astronomical Journal, vol. 128, no. 5, pp. 2170-2205, 2004.

[111] K. A. Venn, M. Irwin, M. D. Shetrone, C. A. Tout, V. Hill, and E. Tolstoy, "Stellar chemical signatures and hierarchical galaxy formation," The Astronomical Journal, vol. 128, no. 3, pp. 1177-1195, 2004.

[112] A. Helmi, M. J. Irwin, E. Tolstoy, et al., "A new view of the dwarf spheroidal satellites of the milky way from VLT FLAMES: where are the very metal-poor stars?" The Astrophysical Journal, vol. 651, pp. L121-L124, 2006.

[113] T. Schoerck, N. Christlieb, J. G. Cohen, et al., "The stellar content of the Hamburg/ESO survey. V. The metallicity distribution function of the Galactic halo," http://arxiv.org/abs/0809.1172.

[114] D. Carollo, T. C. Beers, Y. S. Lee, et al., "Two stellar components in the halo of the Milky Way," Nature, vol. 450, no. 7172, pp. 1020-1025, 2007.

[115] R. G. Gratton, E. Carretta, S. Desidera, S. Lucatello, P. Mazzei, and M. Barbieri, "Abundances for metal-poor stars with accurate parallaxes. II. alpha -elements in the halo," Astronomy \& Astrophysics, vol. 406, pp. 131-140, 2003.

[116] I. U. Roederer, "Chemical inhomogeneities in the milky way stellar halo," The Astronomical Journal, vol. 137, no. 1, pp. 272-295, 2009.

[117] E. N. Kirby, J. D. Simon, M. Geha, P. Guhathakurta, and A. Frebel, "Uncovering extremely metal-poor stars in the Milky way's ultrafaint dwarf spheroidal satellite galaxies," The Astrophysical Journal, vol. 685, no. 1, part 2, pp. L43-L46, 2008.

[118] A. Koch, A. McWilliam, E. K. Grebel, D. B. Zucker, and V. Belokurov, "The highly unusual chemical composition of the hercules dwarf spheroidal galaxy," The Astrophysical Journal, vol. 687, no. 1, part 2, pp. L13-L16, 2008.

[119] A. Frebel, J. D. Simon, M. Geha, and B. Willman, "Highresolution spectroscopy of extremely metal-poor stars in the least evolved galaxies: ursa major II and coma berenices," http://arxiv.org/abs/0902.2395. 

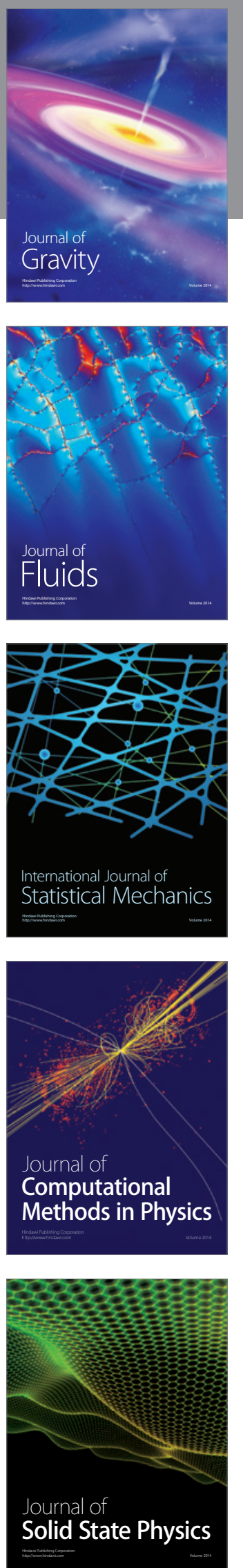

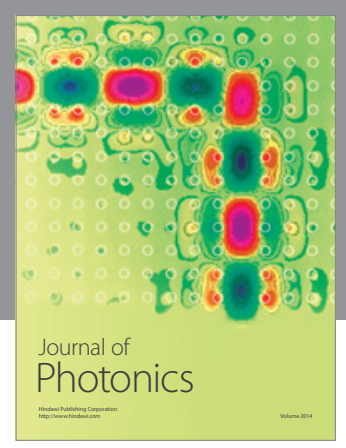

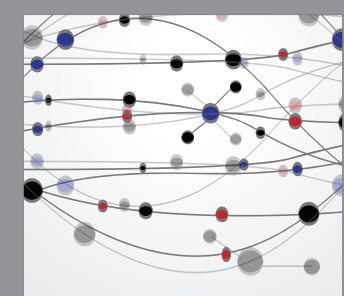

The Scientific World Journal
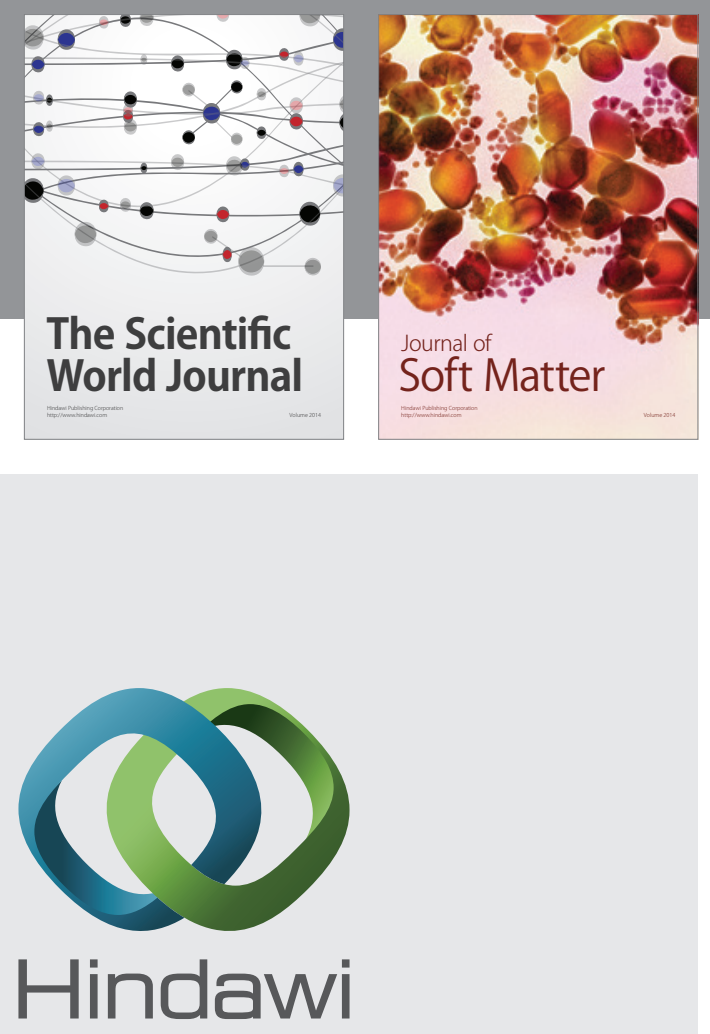

Submit your manuscripts at

http://www.hindawi.com
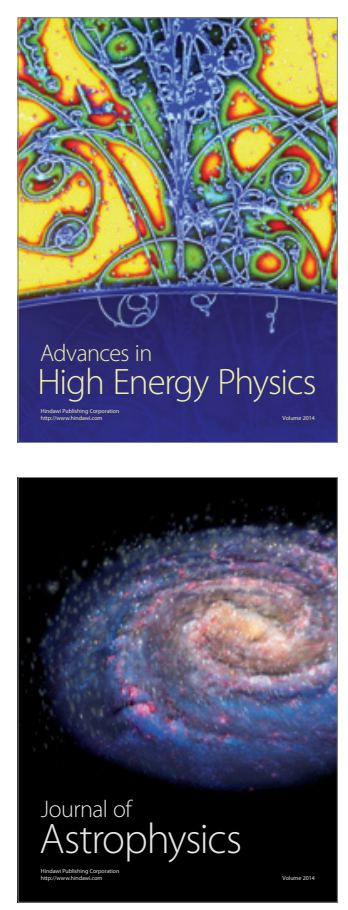
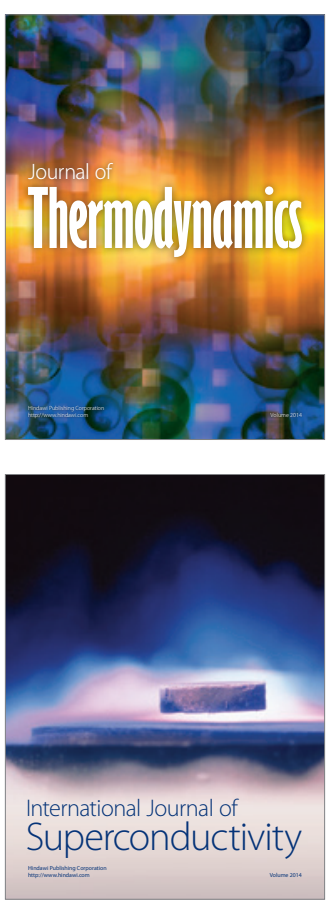
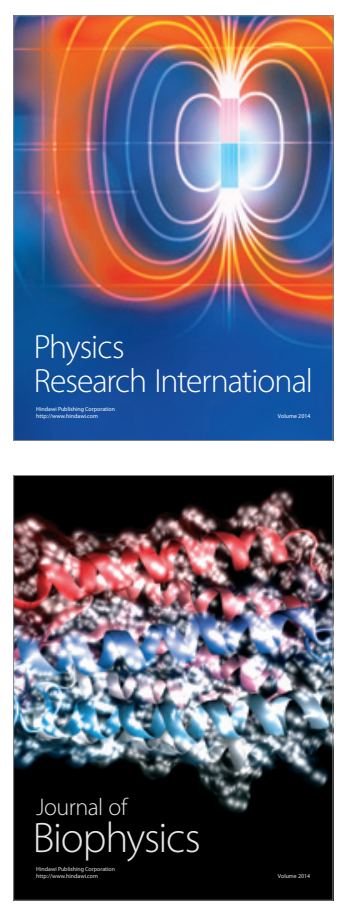
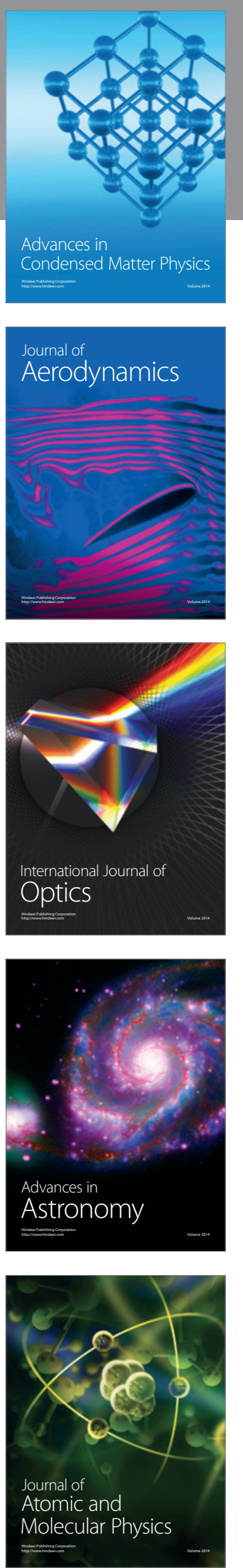